\title{
Vibration reduction of a quadrotor with a cable-suspended payload using polynomial trajectories
}

\author{
Hassan Alkomy $\mathbb{D} \cdot$ Jinjun Shan $\mathbb{D}$
}

Received: 21 December 2020 / Accepted: 12 April 2021 / Published online: 5 May 2021

(C) The Author(s), under exclusive licence to Springer Nature B.V. 2021

\begin{abstract}
This paper considers a transportation system consisting of a quadrotor with a cable-suspended payload. The main focus of this paper is to investigate the effect of polynomial trajectories on the vibration of the cable-suspended payload and to show which polynomial trajectory results in less vibration. A mathematical analysis and a parametric study were carried out to investigate the effect of the degree of the polynomial trajectory on its kinematic behavior. A conjecture relates the degree of the polynomial trajectory and its kinematic behavior to the corresponding payload vibration was introduced. The base excitation model of vibratory systems was proposed as the model of the transportation system of interest. The vibration analysis of both the transportation system and the polynomial trajectories was conducted analytically to show which polynomial trajectory has the least payload vibration. A second stage of payload vibration reduction was provided by introducing a method to reduce the transmitted vibration from the quadrotor to the payload for any quadrotor trajectory. A roadmap to design the transportation task that can reduce the payload vibration was proposed. Both the simulation and the experimental results were presented, discussed and analyzed to verify the findings of this paper.
\end{abstract}

H. Alkomy · J. Shan $(\varangle)$

Department of Earth and Space Science and Engineering,

York University, 4700 Keele St, Toronto M3J 1P3, Canada e-mail: jjshan@yorku.ca

H. Alkomy

e-mail: hmalkomy@yorku.ca
Keywords Quadrotor · Cable-suspended payload . Polynomial trajectory · Vibration · Minimum jerk trajectory

\section{Introduction}

Unmanned aerial vehicles (UAVs) are considered to be one of the revolutionary inventions. They changed, and will change, our lifestyle as they have a wide range of applications that varies from military applications to even entertainment and children toys. One of these important applications is using the UAVs in transportation, e.g., package delivery [1-6]. In these publications, many advantages can be found; however, it becomes more vital in pandemic times, such as COVID-19, since it provides a means for contactless delivery. Generally speaking, the transportation task using a UAV can be categorized into two main categories: grasped payload transportation and cable-suspended payload transportation $[7,8]$. For the grasped payload transportation method, the payload is rigidly attached to the UAV. The resultant system can be treated, dynamically, as a new UAV with a larger mass, different dimensions, and a new center of mass [7-10]. As for the second transportation method, which grasped our attention, the payload is attached to the UAV through a cable, or cables. In contrast with the grasped payload method, the cable-suspended payload method adds underactuated degrees of freedom (DoFs) to the dynamics model, which makes the dynamics of the transportation system 
more complex. Additionally, the vibration of the cablesuspended payload is a big challenge that requires a lot of effort to resolve [8]. In addition to the danger of carrying a vibrating payload, the vibration may add some excessive force components and moments to the system, which can lead to undesired motions.

There are mainly two approaches to reduce the payload vibration: (i) anti-swing control and (ii) trajectory design. The anti-swing controllers, in the context of this paper, are the controllers that aim at reducing or eliminating the payload vibration. Several controllers have been designed for this purpose, such as iterative linear quadratic regulator (iLQR) [11], energy-based adaptive nonlinear anti-swing controllers [12,13], nonlinear model predictive controller (NMPC) [14], input shaping [4], and many other controllers [15-17].

This paper focuses on designing trajectories that can reduce the payload vibration during transportation. In [18], a dynamic programming approach was used to generate a trajectory that is swing-free. In [19], an online trajectory generation approach was used to design an acceleration trajectory, which eliminated the payload vibration. In [20], the authors used the framework of optimal control to generate trajectories for different tasks. The differential flatness of the quadrotor was used in trajectory generation in [21-23]. In [21], the authors found that part of the attitude control inputs are functions of the snap, i.e., the 4th time derivative of the position. Consequently, they designed a trajectory that minimized the snap assuming that this may minimize the control input. This approach was extended in [24] to consider the collision avoidance in cluttered environments. In [25], a polynomial trajectory that minimized the pop, i.e., the 6th time derivative of the position, was generated. A minimum time trajectory was designed, while considering reducing the swing motion in [26]. In [27], a real-time predictive control trajectory considering the shortest route with reduced payload vibration and obstacle avoidance was proposed. Reinforcement learning was applied to generate trajectories that reduce the cable vibration in $[28,29]$. In [30], the trajectory tracking problem for quadcopters was discussed considering the external disturbances and less sensors. In [31], an optimal model-free back stepping controller has been developed for the quadcopter. Implementing this control approach with a cuckoo search algorithm dealt with the uncertainties of the system dynamics and the external disturbances. A very similar dynamics system is the gantry crane. One of the popular ways to reduce the residual payload vibration is to apply the input shaping techniques, which was used in quadrotors in [4] and in gantry cranes in $[32,33]$.

In this paper, the polynomial trajectories, as an important family of trajectories, are studied and their effects on the suspended payload vibration are investigated. One of the advantages of the polynomial trajectories is that they allow for setting any boundary conditions on the trajectory straightforwardly. They also allow for any required degree of smoothness since increasing the degree of the polynomial trajectory increases the smoothness of the trajectory. Additionally, they have a wide range of practical applications other than the aforementioned applications. The minimum jerk trajectory was considered as a good fit of the human arm motions [34], while it was used to reduce the cutting tool vibration in computer numerical control (CNC) machines [35], reduce the cycle time for the laser drilling [36], and, widely, in planning a smooth robot arm motion [37].

Despite the huge potential of the polynomial trajectories, they are rarely addressed in the literature related to the transportation using UAVs. Additionally, most of the related literature considered the $2 \mathrm{D}$ motion and/or lacked the experimental validation. Most importantly, to the best of our knowledge, the literature lacks a substantial investigatory study to show which polynomial trajectory would result in the best vibration behavior. This paper offers this investigatory study and a simpler and more straightforward alternative to the anti-swing controllers to reduce the payload vibration. Specifically, this paper has the following contributions:

- To the best of our knowledge, this paper conducts the first substantial vibration analysis for both the transportation system and the polynomial trajectory to show analytically which polynomial trajectory results in less payload vibration. The paper proposes the base excitation vibration model to represent and analyze the transportation system (Sects. 4.1, 4.2 and 5).

- This paper introduces a method to provide a second stage of payload vibration reduction by reducing the transmitted vibration from the quadrotor to the suspended payload regardless of the type of the quadrotor trajectory. Based on this method, a roadmap to design a transportation task that can 


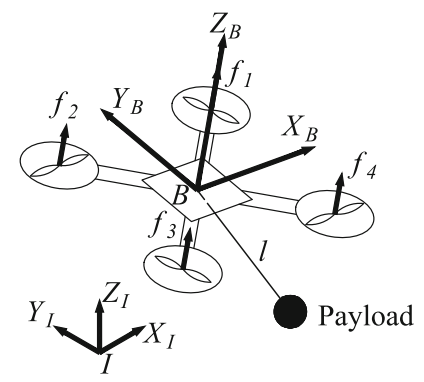

(a) Transportation system

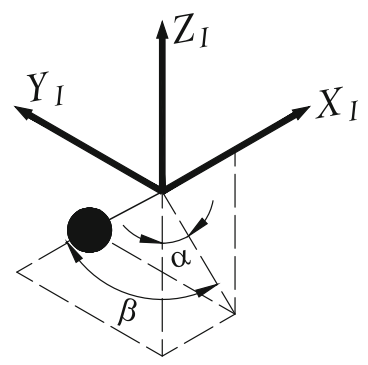

(b) Cable angle

Fig. 1 Schematic of the transportation system and the cable angle

reduce the suspended payload vibration is proposed (Sects. 4.3 and 5).

This paper is organized as follows. The dynamics model is derived and the controller is presented in Sect. 2. Section 3 introduces the polynomial trajectories and their kinematic behavior. The vibration analysis of the transportation system and the polynomial trajectories is conducted in Sect. 4. The simulation results and the experimental validation are presented and discussed in Sects. 5 and 6, respectively. The conclusions are presented in Sect. 7.

\section{Dynamics model}

The transportation system of interest is shown in Fig. 1a, while Fig. 1b shows the cable angles. Two coordinate frames are defined to derive the dynamics model of the transportation system: the inertial frame $\mathcal{I}=\left(X_{I}, Y_{I}, Z_{I}\right)$ and the body-fixed moving frame $\mathcal{B}=\left(X_{B}, Y_{B}, Z_{B}\right)$, which is attached to the quadrotor's center of mass as shown in Fig. 1a. The payload is attached to the quadrotor through a cable of length $l$. The magnitudes of the propellers' thrusts in the direction of $Z_{B}$ are $f_{1}, f_{2}, f_{3}$ and $f_{4}$ at the center of mass of each propeller, respectively. The resultant of these four thrusts is the total thrust $\mathbf{Q}$ in the direction of $Z_{B}$ at the centroid of the quadrotor. Throughout this paper, a point mass payload has been assumed. The center of mass of the quadrotor is the centroid of the quadrotor, and the cable is attached to this center of mass. The cable is non-deformable, non-stretchable, massless and always under tension. Although the center of mass may be either difficult to be accurately specified or not practical to attach the cable to, the assumption of attaching the cable to the center of mass is still reasonable because the offset is considerably small [38]. However, the interested readers can find the dynamics models that consider the offset between the center of mass and the attaching point in [39] and [40].

The transportation system has two subsystems, i.e., a quadrotor system and a 3D pendulum system, which represents the suspended payload. The quadrotor alone is an underactuated system, since it has four actuators (propellers) and six degrees of freedom (DoFs). The addition of the cable-suspended payload adds two more DoFs in case of a taut cable scenario, which is the case of concern in this paper. In addition to the underactuation of the system, the dynamics of the system is highly coupled and nonlinear. Let the positions of the quadrotor and the payload in $\mathcal{I}$ be $\mathbf{q}_{\mathbf{Q}} \in \mathbb{R}^{3}$ and $\mathbf{q}_{\mathbf{L}} \in \mathbb{R}^{3}$, respectively. The inertia matrix of the quadrotor about the quadrotor's center of mass in $\mathcal{B}$ is $\mathbf{I}_{\mathbf{Q}} \in \mathbb{R}^{3 \times 3}$ and the angular velocity of the quadrotor in $\mathcal{B}$ is $\omega_{\mathbf{Q}} \in \mathbb{R}^{3}$. Let $\mathbf{e} \in \mathbb{R}^{3}$ be a unit vector in the direction of $Z_{I}$ expressed in the inertial frame $\mathcal{I}$, and $m_{Q}$ and $m_{L}$, are all in $\mathbb{R}_{>0}$, and they represent the mass of the quadrotor and the payload, respectively. Following the Lagrangian method, under the assumptions mentioned above, to find the dynamics model of the transportation system, the Lagrangian, $\mathcal{L}$, can be defined as

$$
\begin{aligned}
\mathcal{L} & =\frac{1}{2} m_{Q}\left(\dot{\mathbf{q}}_{\mathbf{Q}} \cdot \dot{\mathbf{q}}_{\mathbf{Q}}\right)+\frac{1}{2} m_{L}\left(\dot{\mathbf{q}}_{\mathbf{L}} \cdot \dot{\mathbf{q}}_{\mathbf{L}}\right) \\
& +\frac{1}{2} \mathbf{I}_{\mathbf{Q}}(\boldsymbol{\omega} \cdot \boldsymbol{\omega}) \\
& -m_{Q} g\left(\mathbf{q}_{\mathbf{Q}} \cdot \mathbf{e}\right)-m_{L} g\left(\mathbf{q}_{\mathbf{L}} \cdot \mathbf{e}\right)
\end{aligned}
$$

Since the cable is attached to the center of mass of the quadrotor, the position of the payload can be expressed in terms of the position of the quadrotor and $\mathbf{r}_{\mathbf{m}} \in \mathbb{R}^{3}$, where $\mathbf{r}_{\mathbf{m}} \in \mathbb{R}^{3}$ is a unit vector from the origin of the body fixed frame, $\mathcal{B}$, to the payload expressed in the inertial frame $\mathcal{I}$, as follows

$\mathbf{q}_{\mathbf{L}}=\mathbf{q}_{\mathbf{Q}}+l \mathbf{r}_{\mathbf{m}}$ 
From Eqs. (1) and (2), the Lagrangian $\mathcal{L}$ can be expressed as

$$
\begin{aligned}
\mathcal{L}= & \frac{1}{2}\left(m_{Q}+m_{L}\right)\left(\dot{\mathbf{q}}_{\mathbf{Q}} \cdot \dot{\mathbf{q}}_{\mathbf{Q}}\right)+\frac{1}{2} m_{L} l^{2}\left(\dot{\mathbf{r}}_{\mathbf{m}} \cdot \dot{\mathbf{r}}_{\mathbf{m}}\right) \\
& +m_{L} l\left(\dot{\mathbf{q}}_{\mathbf{Q}} \cdot \dot{\mathbf{r}}_{\mathbf{m}}\right)+\frac{1}{2} \mathbf{I}_{\mathbf{Q}}(\boldsymbol{\omega} \cdot \boldsymbol{\omega}) \\
& -\left(m_{Q}+m_{L}\right) g(\mathbf{q} \mathbf{Q} \cdot \mathbf{e})-m_{L} g l\left(\mathbf{r}_{\mathbf{m}} \cdot \mathbf{e}\right)
\end{aligned}
$$

Now, let $\left(\boldsymbol{\eta}=[\alpha, \beta]^{\top} \in \mathbb{R}^{2}\right)$ be the vector that includes the angles of the cable, as shown in Fig. $1 \mathrm{~b}$, then $\mathbf{r}_{\mathbf{m}}$ can be defined as $\mathbf{r}_{\mathbf{m}}=[\sin \alpha \cos \beta, \sin \beta$, $-\cos \alpha \cos \beta]^{\top}$. The time derivatives of $\mathbf{r}_{\mathbf{m}}$ can be expressed in terms of the angles of the cable as $\dot{\mathbf{r}}_{\mathbf{m}}=\mathbf{A} \dot{\eta}$ and $\ddot{\mathbf{r}}_{\mathbf{m}}=\mathbf{B} \dot{\eta}+\mathbf{A} \ddot{\eta}$, where $\mathbf{A}=$ $[\cos \alpha \cos \beta-\sin \alpha \sin \beta]$

$\left[\begin{array}{cc}0 & \cos \beta \\ \sin \alpha \cos \beta & \cos \alpha \sin \beta\end{array}\right]$ and $\mathbf{B}=\dot{\mathbf{A}}$.

Before obtaining the dynamics model of the transportation system, some vectors and matrices need to be defined. For the translation motion of the transportation system, the generalized coordinate vector is $\mathbf{q}=\left[\mathbf{q}_{\mathbf{Q}}, \boldsymbol{\eta}\right]^{\top} \in \mathbb{R}^{5}$. The inertia matrix is $\mathbf{M}=$ $\left[\begin{array}{ll}\mathbf{M}_{11} & \mathbf{M}_{12} \\ \mathbf{M}_{21} & \mathbf{M}_{22}\end{array}\right] \in \mathbb{R}^{5 \times 5}$, where $\mathbf{M}_{11}=\operatorname{diag}\left(m_{Q}+m_{L}\right) \in$ $\mathbb{R}^{3 \times 3}, \mathbf{M}_{\mathbf{2 2}}=\left[m_{L} l^{2} \mathbf{A}^{\top} \mathbf{A}\right] \in \mathbb{R}^{2 \times 2}, \mathbf{M}_{\mathbf{1 2}}=\mathbf{M}_{\mathbf{2 1}}^{\top}=$ $\left[m_{L} l \mathbf{A}\right] \in \mathbb{R}^{3 \times 2}$. The damping and the gravitational matrices are $\mathbf{C}=\left[\begin{array}{ll}\mathbf{0}_{5 \times \mathbf{3}} & \mathbf{C}_{\mathbf{1 2}}\end{array}\right] \in \mathbb{R}^{5 \times 5}$, with $\mathbf{C}_{\mathbf{1 2}}=$ $\left[\begin{array}{c}m_{L} l \mathbf{B} \\ m_{L} l^{2} \mathbf{A}^{\top} \mathbf{B}\end{array}\right] \in \mathbb{R}^{5 \times 2}$, and $\mathbf{G}=\left[\begin{array}{c}\left(m_{Q}+m_{L}\right) g \mathbf{e} \\ m_{L} g l \mathbf{A}^{\top} \mathbf{e}\end{array}\right] \in$ $\mathbb{R}^{5}$, respectively. The total thrust vector in the three inertial directions is $\mathbf{Q}=\left[Q_{x}, Q_{y}, Q_{z}\right]^{\top} \in \mathbb{R}^{3}$, so the generalized force vector is $\mathbf{F}=[\mathbf{Q}, 0,0]^{\top} \in \mathbb{R}^{5}$. For the rotational motion, the generalized moment vector is $\boldsymbol{\tau}=\left[\tau_{x}, \tau_{y}, \tau_{z}\right]^{\top} \in \mathbb{R}^{3}$.

Now, the dynamics model of the transportation system can be obtained from Eq. (3), after substituting $\mathbf{r}_{\mathbf{m}}$ and its time derivatives, as

$$
\begin{aligned}
& \mathbf{M} \ddot{\mathbf{q}}+\mathbf{C} \dot{\mathbf{q}}+\mathbf{G}=\mathbf{F} \\
& \mathbf{I}_{\mathbf{Q}} \dot{\omega}_{\mathbf{Q}}+\omega_{\mathbf{Q}} \times \mathbf{I}_{\mathbf{Q}} \omega_{\mathbf{Q}}=\tau
\end{aligned}
$$

Note that $\mathbf{Q}$ represents the total thrust and $\boldsymbol{\tau}$ is the generalized moment. The total thrust $\mathbf{Q}$ is function of the attitude angles of the quadrotor, so Eqs. (4) and (5) are coupled. However, this paper focuses on the translational motion of the transportation system, i.e., Eq. (4). Consequently, there is no need to deal with the attitude dynamics at this moment. This paper deals with the total thrust $\mathbf{Q}$ as a force with three components $Q_{x}, Q_{y}$ and $Q z$ without expressing these components in terms

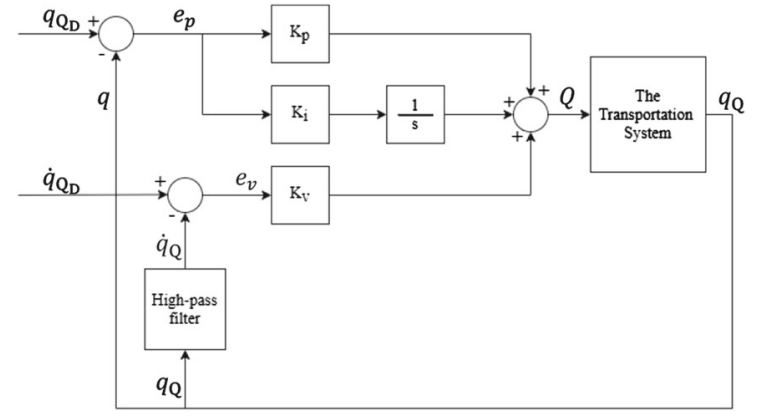

Fig. 2 The PIV controller

of the attitude angles. It is worth noting that the total thrust $\mathbf{Q}$ is expressed in the inertial frame $\mathcal{I}$.

A proportional-integral-velocity (PIV) controller is designed based on feeding back the quadrotor's measurements. The feedback of the suspended payload measurements is not used in controlling the motion. The main difference between the PIV controller and the conventional proportional-integral-derivative (PID) controller comes from the definition of the error. In the PID, there is only the position error $\mathbf{e}_{\mathbf{p}}$. In the PIV, however, there are the position and velocity errors, $\mathbf{e}_{\mathbf{p}}$ and $\mathbf{e}_{\mathbf{v}}$, respectively as shown in Fig. 2. The velocity error $\mathbf{e}_{\mathbf{v}}$ is calculated as the difference between the desired velocity and the actual velocity (not the time derivative of $\mathbf{e}_{\mathbf{p}}$ ). Since the direct time derivative of $\mathbf{e}_{\mathbf{p}}$ is not taken in the PIV, the control output is less noisy and the closed-loop response has less overshoot. Since the focus of this paper is on the translational motion, the position PIV controller can be defined as follows

$\mathbf{Q}=\mathbf{K}_{\mathbf{p}} \mathbf{e}_{\mathbf{p}}+\mathbf{K}_{\mathbf{v}} \mathbf{e}_{\mathbf{v}}+\mathbf{K}_{\mathbf{i}} \mathbf{e}_{\mathbf{i}}$

where, the control gains matrices are $\mathbf{K}_{\mathbf{p}}=\operatorname{diag}\left(\frac{\pi}{2}, \frac{\pi}{2}\right.$, 35) $\in \mathbb{R}^{3 \times 3}, \mathbf{K}_{\mathbf{v}}=\operatorname{diag}\left(\frac{\pi}{4}, \frac{\pi}{4}, 14\right) \in \mathbb{R}^{3 \times 3}$, and $\mathbf{K}_{\mathbf{i}}=$ $\operatorname{diag}(0,0,4) \in \mathbb{R}^{3 \times 3}$. The errors can be defined as $\mathbf{e}_{\mathbf{p}}=\mathbf{q}_{\mathbf{Q}_{\mathbf{D}}}-\mathbf{q}_{\mathbf{Q}}, \mathbf{e}_{\mathbf{v}}=\dot{\mathbf{q}}_{\mathbf{Q}_{\mathrm{D}}}-\dot{\mathbf{q}}_{\mathbf{Q}}$, and $\dot{\mathbf{e}}_{\mathbf{i}}=\mathbf{e}_{\mathbf{p}}$, where the subscript $\mathbf{D}$ represents the desired trajectory.

\section{The polynomial trajectories}

\subsection{Generation of polynomial trajectories}

One of the main objectives of this paper is to investigate the effect of the polynomial trajectories on the suspended payload vibration. The polynomial trajectories have the following general form in one dimension 
$x=\sum_{i=0}^{j} c_{i} t^{i}$

where $x$ is a polynomial trajectory of the $j$ th degree, $c_{i}$ is the coefficient of the $i$ th term, and $t$ is the time.

The higher the degree of the polynomial, the smoother the trajectory becomes. However, the smoothness is not the only objective of the trajectory. It is a good idea to minimize a cost function that has an effect on the performance of the transportation task. For example in [21], the authors found that part of the attitude control input is a function of the fourth time derivative of the position of the quadrotor; then, they made a conjecture that minimizing the fourth time derivative of the position, i.e., the snap, may lead to minimizing the control input. In [25], the authors minimized the 6th time derivative of the position as they found that the highest time derivative in the input is the 6th. There is no solid criteria found in the literature to choose which quantity needs to be minimized to obtain the best performance. Consequently, an investigation is required. So, it is important to present a method to design a trajectory that minimizes any quantity in general.

In Eq. (7), the degree of the polynomial and some or all of the coefficients, $c_{i}$, can be chosen arbitrarily or to satisfy certain specifications. If there are more constraints and specifications that the trajectory has to satisfy, the degree of the polynomial gets higher. While designing trajectories, some cost functions may be required to be minimized. For example, the trajectory may be required to minimize the acceleration, the jerk, the snap or any other cost function. The resultant trajectories are called minimum acceleration, minimum jerk or minimum snap trajectories, respectively. In general, the trajectory that minimizes any of the time derivatives of the position function, $x_{\min }$, can be found as follows

$x_{\min }=\arg \min _{x(t)} \int_{0}^{t}\left(\frac{d^{k} x(t)}{\mathrm{d} t^{k}}\right)^{2} \mathrm{~d} t$

where $k$ is the order of the position time derivative that is required to be minimized by the trajectory, $x_{\min }$, e.g., $k=2$ for the minimum acceleration trajectory.

For the sake of generalization, a functional $L=$ $\left(\frac{d^{k} x(t)}{\mathrm{d} t^{k}}\right)^{2}$ is defined, then the Euler-Lagrange solution to the optimization problem in Eq. (8) can be found as follows $\frac{\partial L}{\partial x}-\frac{d}{\mathrm{~d} t} \frac{\partial L}{\partial \dot{x}}+\cdots+(-1)^{k} \frac{d^{k}}{\mathrm{~d} t^{k}} \frac{\partial L}{\partial x^{(k)}}=0$

where $x^{(k)}$ is the $k$ th time derivative of $x$ and $k$ is defined as in Eq. (8).

For UAV transportation applications, usually the transportation task ends with a complete stop of the UAV. This means ending at zero velocity and zero acceleration, which means that there are constraints on the velocity and acceleration. This omits the minimum velocity and the minimum acceleration trajectories from the potential trajectories set. In this paper, three different polynomial trajectories are discussed, namely the minimum jerk trajectory (MJT), the minimum snap trajectory (MST) and the minimum crackle trajectory (MCT).

Based on Eq. (9), the MJT, the MST and the MCT are on the forms of

$$
\begin{aligned}
& x_{j}=\sum_{i=0}^{5} c_{i} t^{i} \\
& x_{s}=\sum_{i=0}^{7} c_{i} t^{i} \\
& x_{c}=\sum_{i=0}^{9} c_{i} t^{i}
\end{aligned}
$$

The polynomial trajectory in Eq. (7) can be defined by specifying the coefficients, $c_{i}$, using the trajectory specifications and constraints, i.e., the boundary conditions. These coefficients can be specified by rewriting Eq. (7) as follows

$\mathbf{C}=\mathbf{T}^{-1} \mathbf{S}$

where $\mathbf{C}=\left[c_{j}, c_{j-1}, \ldots, c_{0}\right]^{\top} \in \mathbb{R}^{j+1}$ is the coefficients vector that contains all the polynomial coefficients, $\mathbf{S}=\left[x_{0}, x_{f}, \dot{x}_{0}, \dot{x}_{f}, \ddot{x}_{0}, \ddot{x}_{f}, \ldots, x_{0}^{\left(\frac{j-1}{2}\right)}\right.$, $\left.x_{f}^{\left(\frac{j-1}{2}\right)}\right]^{\top} \in \mathbb{R}^{j+1}$ is the specifications vector, which contains the boundary conditions, and (.) $\left(\frac{j-1}{2}\right)$ is the $\left(\frac{j-1}{2}\right)^{t h}$ position time derivative constraint. The subscript 0 in the position $x$ and its time derivatives refers to the correspondence with the initial point, whereas the 


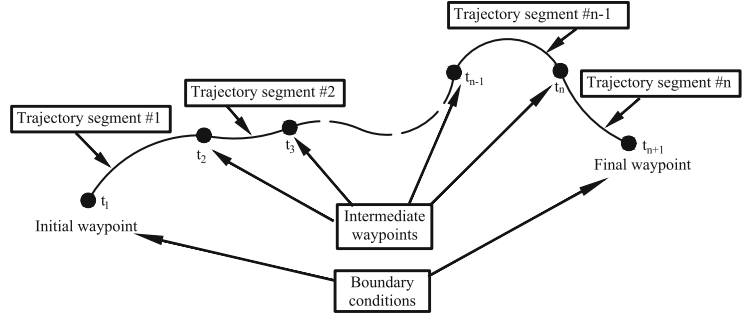

Fig. 3 A general representation of a multi-segment polynomial trajectory

subscript $f$ refers to the final point. The timing matrix, $\mathbf{T} \in \mathbb{R}_{\geq 0}^{(j+1) \times(j+1)}$ can be formed as follows

$\mathbf{T}=\left[\begin{array}{ccccc}t_{0}^{j} & t_{0}^{j-1} & \cdots & t_{0} & 1 \\ t_{f}^{j} & t_{f}^{j-1} & \cdots & t_{f} & 1 \\ j t_{0}^{j-1} & (j-1) t_{0}^{j-2} & \cdots & 1 & 0 \\ j t_{f}^{j-1} & (j-1) t_{f}^{j-2} & \cdots & 1 & 0 \\ \vdots & \vdots & \vdots & \vdots & \vdots \\ j(j-1) \ldots\left(\frac{j+3}{2}\right) t_{0}^{\left(\frac{j+1}{2}\right)} & \cdots & \cdots & \cdots & 0 \\ j(j-1) \ldots\left(\frac{j+3}{2}\right) t_{f}^{\left(\frac{j+1}{2}\right)} & \cdots & \cdots & \cdots & 0\end{array}\right]$

where, $t_{0}$ and $t_{f}$ are the initial and final times, respectively.

\subsection{Multi-segment polynomial trajectory generation}

The polynomial trajectory expressed by Eq. (7) represents a trajectory between two waypoints, i.e., initial and final points only. If multiple waypoints are required to be passed through by the polynomial trajectory, the trajectory in such a case should be a piecewise function depending on the number of the required waypoints. Let $n+1$ be the number of waypoints, which means that $n$ is the number of the trajectory segments or the number of the pieces of the piecewise function. A trajectory segment is the trajectory between any two waypoints. The time vector that represents the desired times of the waypoints is $\mathbf{T}_{\mathbf{D}}=\left[t_{1}, t_{2}, t_{3}, \ldots, t_{n+1}\right]^{\top} \in \mathbb{R}_{\geq 0}^{n+1}$. Figure 3 pictates a general multi-segment polynomial trajectory. Since each segment has its own trajectory, there are $n$ sets of the coefficients $c_{i}$. These different sets will be referred to as $c_{i, p}$, where $p$ is the number of the trajectory segment, i.e., the number of pieces of the piecewise function, and $p \in\{1,2, \ldots, n\}$. A general formula of a multi-segment polynomial trajectory, i.e., multiple waypoints trajectory, can be expressed as

$$
x= \begin{cases}\sum_{i=0}^{j} c_{i, 1} t^{i}, & t_{1} \leq t \leq t_{2} \\ \sum_{i=0}^{j} c_{i, 2} t^{i}, & t_{2}<t \leq t_{3} \\ \sum_{i=0}^{j} c_{i, 3} t^{i}, & t_{3}<t \leq t_{4} \\ \cdots & \\ \sum_{i=0}^{j} c_{i, n} t^{i}, & t_{n}<t \leq t_{n+1}\end{cases}
$$

Consider a multi-segment polynomial trajectory, as in Eq. (12) and Fig. 3, with $n$ segments and of $j^{\text {th }}$ degree. The number of the required conditions to fully determine the trajectory is $(j+1) n$. Although there are many ways to set these conditions based on the practical applications, the available information and/or the desired specifications, this paper is restricted to one of these ways. Throughout this paper, the boundary conditions at all of the waypoints are assumed to be known. The boundary conditions here mean the positions of the waypoints, and their time derivatives up to the $\left(\frac{j-1}{2}\right)^{t h}$ time derivative. In this case, to solve for the trajectory, i.e., finding the coefficients from Eq. (11) and the trajectory from Eq. (12), the matrices in Eq. (11) need to be redefined. The specifications vector, $\mathbf{S} \in \mathbb{R}^{n(j+1)}$, can be expressed as

$$
\begin{aligned}
\mathbf{S}= & {\left[x_{1}, x_{2}, x_{2}, x_{3}, x_{3}, \ldots, x_{n}, x_{n}, x_{n+1} \mid\right.} \\
& \left.\dot{x}_{1}, \dot{x}_{2}, \dot{x}_{2}, \dot{x}_{3}, \dot{x}_{3}, \ldots, \dot{x}_{n}, \dot{x}_{n}, \dot{x}_{n+1} \mid \cdots\right]^{\top}
\end{aligned}
$$

and the time derivatives follow the same pattern up to the $\left(\frac{j-1}{2}\right)^{t h}$ time derivative.

The timing matrix $\mathbf{T} \in \mathbb{R}^{n(j+1) \times n(j+1)}$ can be expressed as follows 


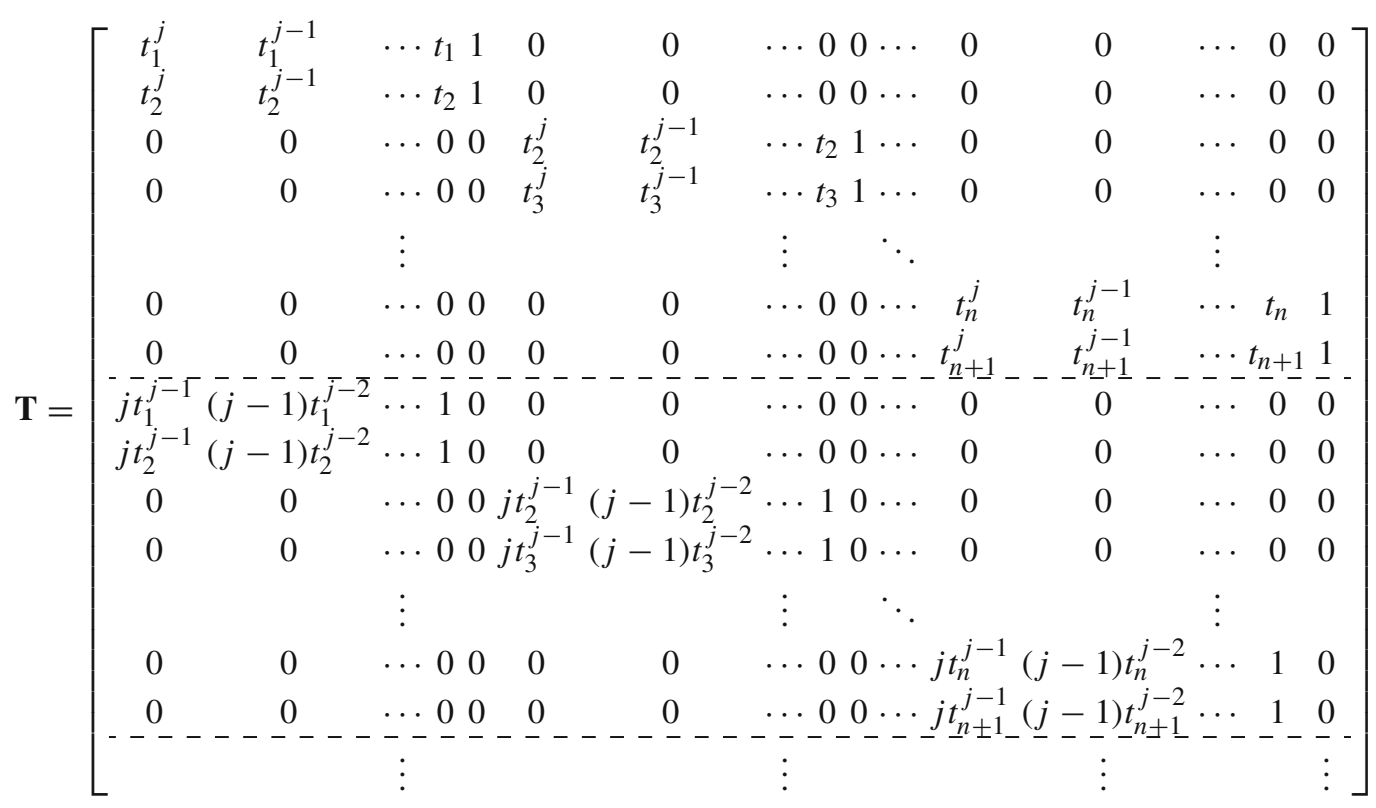

The timing matrix in Eq. (14) has $\frac{j+1}{2}$ horizontal partitions, in which, the the upper partition of the matrix represents the positions of all of the waypoints. The second partition is for the first position time derivatives, i.e., the velocities, of all of the waypoints. The third partition is for the second position time derivatives of all of the waypoints and so on so forth up to the $\left(\frac{j-1}{2}\right)^{t h}$ time derivative. Solving Eq. (11) results in finding the coefficients vector, $\mathbf{C}=\left[c_{j, 1}, c_{j-1,1}, \ldots, c_{0,1}, c_{j, 2}, c_{j-1,2}, \ldots, c_{0,2}, \ldots\right.$, $\left.c_{j, n}, c_{j-1, n}, \ldots, c_{0, n}\right]^{\top} \in \mathbb{R}^{n(j+1)}$.

It is worth mentioning that the polynomial trajectory generation process that is presented in Sects. 3.1 and 3.2 has been conducted for a 1D trajectory. Similar procedure can be followed to generate a general 3D polynomial trajectory. The 3D polynomial trajectory, q, can be found as

$\mathbf{q}=[x, y, z]^{\top}$

where $x, y$ and $z$ are defined as multi-segment polynomial trajectories as indicated in Eq. (12).

\subsection{The degree of the polynomial trajectory and its} kinematics

The relation between the degree of the polynomial trajectory and its kinematic behavior is investigated in this subsection. Since this paper investigates the effect of the polynomial trajectories on the suspended payload vibration, we conjectured that the trajectory that has a small rate of change of velocity, i.e., the acceleration, may result in less vibration. Additionally, we conjectured that the trajectory that has small peak velocity and acceleration may also result in less vibrations. Consequently, this subsection focuses, in particular, on the velocity and acceleration profiles of the polynomial trajectories.

In case of the stationary boundary condition, i.e., all of the boundary conditions of the position time derivatives are set to zero, a general formula of the velocity profile can be found as a function of the peak velocity, $v_{p}$, and the relative time, $T_{\text {rel }}=\frac{t}{T_{\text {tot }}}$, as follows [41]

$\left[v=v_{p}\left(4 T_{\text {rel }}\left(1-T_{\text {rel }}\right)\right)^{\frac{j-1}{2}}\right]$

where $T_{\text {tot }}$ is the total time of the trajectory, and the peak velocity, $v_{p}$, can be expressed in terms of the average speed, $v_{\text {avg }}$, as follows

$v_{p}=v_{\mathrm{avg}} \frac{1}{4^{\frac{j-1}{2}}} \frac{j !}{\frac{j-1}{2} !^{2}}$

Equations (16) and (17) show that increasing the degree of the polynomial, $j$, results in increasing the peak velocity, $v_{p}$. 
To examine the kinematic behavior of the polynomial trajectories, a parametric study to see how the degree of the polynomial affects the velocity and acceleration profiles is conducted. The parametric study is designed to simulate a pick-up and drop-off transportation task. Consequently, the stationary boundary conditions are considered. The task is a 3D motion from an initial position $[0,0,0]$ to a final position $[1,1,1]$ and is required to be done in a total time $T_{\text {tot }}=3 \mathrm{~s}$. The parametric study considered the MJT, MST, MCT and the minimum pop trajectory (MPT). Figure 4 pictates the effect of the degree of the polynomial trajectory on the position, velocity and acceleration profiles in a 3D transportation task. Looking at the position profile in Fig. 4, one can notice that increasing the degree of the polynomial trajectory leads the position profile to become more steep and approaches the behavior of the step function. The increase in the slope of the position profile greatly affects the velocity profile as shown in the middle graph in Fig. 4. As the degree of the polynomial trajectory increases, the peak velocity increases and the velocity profile becomes narrower and steeper. This matches with the results of Eqs. (16) and (17) and the results in [41]. Similarly, the peak value of the acceleration increases with the increase in the degree of the polynomial trajectory, and it reaches zero at the middle of the time span, which is corresponding to the turning point in the velocity profile. It is worth mentioning that these graphs are applicable to the motion in any of the three directions, i.e., $x, y$ or $z$ motion. This parametric study shows that the MJT has the lowest peak velocity and acceleration, and its profiles are less steep. Based on our conjecture, the MJT may result in less vibration. However, this conjecture requires to be investigated and proven, which is conducted in Sect. 4.

\section{Vibration analysis}

4.1 The vibration analysis of the transportation system

The translational dynamics model of the transportation system is represented by the five DoFs model shown in Eq. (4), which has five equations describing its motion. The last two equations represent the suspended payload motion, which is the focus of this subsection. They can be expressed as follows

$$
\mathbf{A}^{\top} \ddot{\mathbf{q}} \mathbf{Q}+l \mathbf{A}^{\top} \mathbf{A} \ddot{\boldsymbol{\eta}}+l \mathbf{A}^{\top} \mathbf{B} \dot{\boldsymbol{\eta}}+g \mathbf{A}^{\top} \mathbf{e}=\mathbf{0}
$$

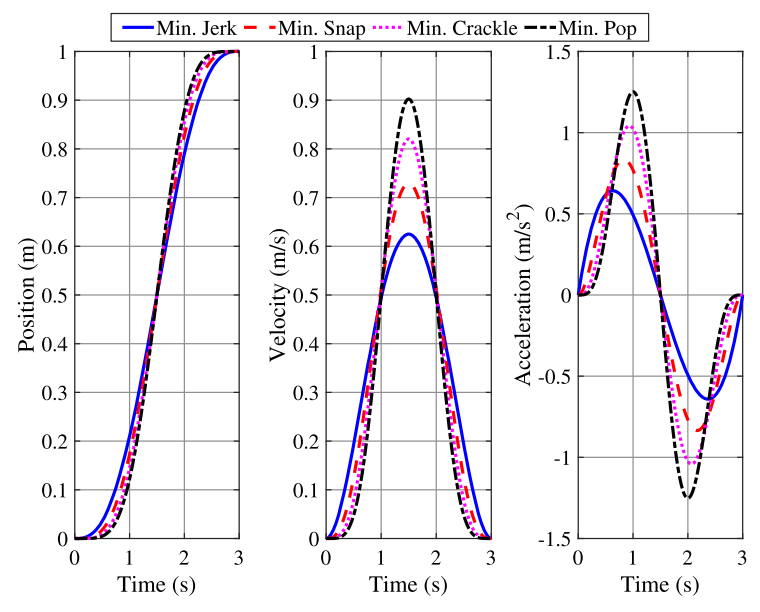

Fig. 4 Position, velocity and acceleration profiles for MJT, MST, MCT and MPT

where $\mathbf{0} \in \mathbb{R}^{2}$ is a vector of zeros and the rest of the parameters and variables are defined as in Eq. (4).

As shown in Eq. (18), the system undergoes nonlinear damped vibrations. In pursuance of determining the natural frequencies and vibration modes of the system, it is acceptable to simplify the system in Eq. (18) by linearizing the system around the suspended payload equilibrium state, i.e., $\boldsymbol{\eta}=0$ and $\dot{\boldsymbol{\eta}}=0$. Under this assumption, the following form of Eq. (18) can be obtained

$\mathbf{M}_{\mathrm{s}} \ddot{\boldsymbol{\eta}}+\mathbf{K}_{\mathrm{s}} \boldsymbol{\eta}=-\ddot{\mathbf{q}}_{\mathbf{Q}_{1,2}}$

where $\mathbf{M}_{\mathbf{s}}=\operatorname{diag}(l) \in \mathbb{R}^{2 \times 2}$ is the simplified inertia matrix, $\mathbf{K}_{\mathbf{s}}=\operatorname{diag}\left(g+\ddot{z}_{Q}\right) \in \mathbb{R}^{2 \times 2}$ is the simplified stiffness matrix, $\mathbf{q Q}_{\mathbf{1}, \mathbf{2}} \in \mathbb{R}^{2}$ is a vector contains the first two elements of the quadrotor's position vector, i.e., $\mathbf{q}_{\mathbf{Q}_{\mathbf{1}, 2}}=\left[x_{Q}, y_{Q}\right]^{\top}$, and $z_{Q}$ is the third element of the the quadrotor's position vector.

Consequently, the natural frequencies and the mode shapes can be found by solving the following eigenvalue problem

$\left[M_{s}^{-1} K_{s}\right] \xi=\omega_{n}^{2} \xi$

where $\omega_{n}$ is the natural frequency of the system and $\xi$ is its corresponding mode shape.

For the system in Eq. (19), the two natural frequencies are

$\omega_{n_{1}}=\omega_{n_{2}}=\sqrt{\frac{g+\ddot{z}_{Q}}{l}}$

and the two vibration mode shapes are

$\xi_{1}=\left[\begin{array}{l}1 \\ 0\end{array}\right], \xi_{2}=\left[\begin{array}{l}0 \\ 1\end{array}\right]$ 
which form a unit modal matrix, $\boldsymbol{\Xi}$, as follows

$$
\boldsymbol{\Xi}=\left[\xi_{1}, \xi_{2}\right]=\left[\begin{array}{ll}
1 & 0 \\
0 & 1
\end{array}\right]
$$

This unit modal matrix shows that the simplified dynamics model in Eq. (19) is decoupled in the states. In fact, this is due to the way of defining the cable angles, $\alpha$ and $\beta$, as shown in Fig. 1b. The system in Eq. (19) is neither completely linear nor completely decoupled because Eq. (18) was not linearized about the input operating point, i.e., the operating point of $\ddot{\mathbf{q}}_{\mathbf{Q}}$. However, the system will be completely linear and decoupled in the states and the inputs if the quadrotor's motion is constrained to have a constant acceleration in the inertial vertical direction $Z_{I}$, e.g., $\ddot{z}_{Q}=0$. In such a case, the system will be a linear time-invariant (LTI) vibratory system. Equation (21) shows that the natural frequency, $\omega_{n}$, increases with the increase in $\ddot{z}_{Q}$, and it is inversely proportional to the square root of the cable length, $\sqrt{l}$. Figure 5a illustrates the effect of the trajectory's vertical acceleration, $\ddot{z}_{Q}$, on the natural frequency, $\omega_{n}$, at different cable length, $l$. It is obvious that the effect of $\ddot{z}_{Q}$ becomes less important when the cable length gets longer. Throughout this paper, the vertical acceleration will be set to $\ddot{z}_{Q}=0$, so Fig. 5b shows the effect of the cable length on the natural frequency for the case of $\ddot{z}_{Q}=0$. Figure $5 \mathrm{~b}$ starts from $l=0.1$ $\mathrm{m}$ because shorter cable lengths will results in higher frequencies, which approaches infinity at $l=0$ based on Eq. (21). Additionally, cables lengths less than 0.1 $\mathrm{m}$ are out of the practical scope.

Before we continue the analysis, it is worth emphasizing two points:

- Dynamically, the input of the system in Eq. (19) is $\ddot{\mathbf{q}}_{\mathbf{Q}_{1,2}}$, while the generalized coordinates vector, i.e., the state vector, is $\boldsymbol{\eta}$.

- Mathematically, the dynamics system in Eq. (19) can be thought of as either a forced vibratory system or a vibratory system with base excitation. In the former description, the excitation force is $\ddot{\mathbf{q}}_{\mathbf{Q}_{1,2}}$, while in the latter description, the base motion follows the trajectory $\mathbf{q}_{\mathbf{Q}_{1,2}}$. Although there is no mathematical difference between either description, thinking of the transportation system as a base excitation model makes more sense because, practically, the payload vibration comes from the motion of the quadrotor, which is the base in the latter description. Thus, throughout this paper, the payload vibration will be dealt with as a base excited

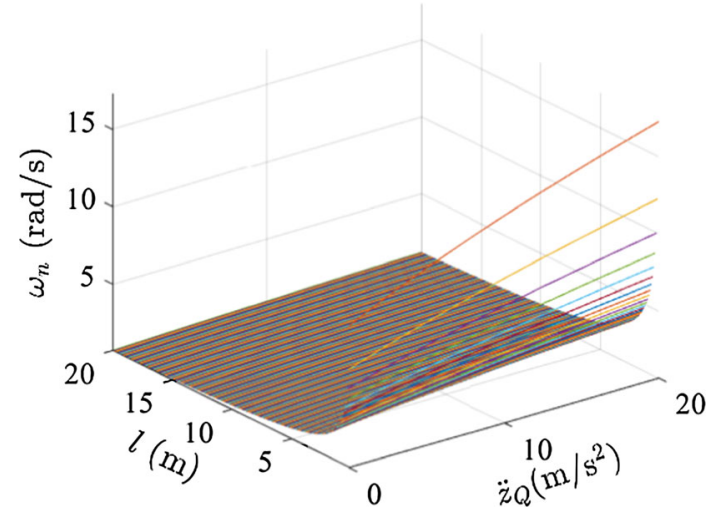

(a) The effect of the trajectory's vertical acceleration, $\ddot{z}_{Q}$, on the natural frequency, $\omega_{n}$, at different cable length, $l$

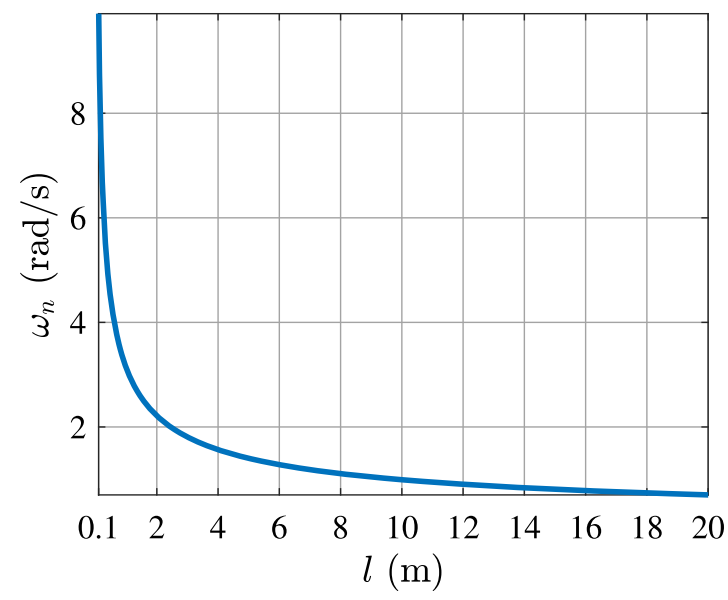

(b) The variation of the natural frequency $\omega_{n}$ with respect to the cable length, $l$, at $\ddot{z}_{Q}=0$

Fig. 5 The variation of the natural frequency of the transportation system

vibration. More details about the vibratory systems with base excitation can be found, for example, in $[42,43]$.

4.2 The vibration analysis of the polynomial trajectories

As shown in Eq. (19), the base excitation frequency is the frequency of the acceleration of the polynomial trajectories. Consequently, a deep frequency analysis of these trajectories is required to determine their frequencies, the magnitudes of these frequencies, and how related they are to the natural frequencies of the trans- 
portation system indicated in Eq. (21). The frequency analysis will be done by obtaining the Fourier series of the accelerations of the trajectories, which represent the base excitation. This way, the trajectories' frequencies and their corresponding magnitudes will be obtained analytically, which allows for further rigorous analysis and comparisons between these trajectories. For the sake of conveying the fundamentals and the logic of the vibration analysis of the polynomial trajectories without getting the readers involved in unnecessary complex mathematics, the very common transportation task with stationary boundary conditions is considered. In other words, the considered transportation task is between two points, where it starts from rest and ends at rest. This implies that all the boundary conditions of the position time derivatives in the specifications vector $\mathbf{S}$ are set to zero. Without loss of generality, the initial time and position are set to zero, i.e., $t_{0}=0$ and $x_{0}=0$. The main equations in this subsection are presented under general boundary conditions in Appendix 1. From there, it is found that the polynomial trajectories show similar vibration behavior under any arbitrary boundary conditions.

The Fourier series of a general function, $f(t)$, which is periodic over the period $\delta$ can be found as an infinite sum of sines and cosines as follows [44]

$$
\begin{aligned}
& f(t)=\frac{a_{0}}{2} \\
& +\sum_{k=1}^{\infty}\left(a_{k} \cos \left(\frac{2 \pi k t}{\delta}\right)+b_{k} \sin \left(\frac{2 \pi k t}{\delta}\right)\right)
\end{aligned}
$$

where

$$
\begin{aligned}
a_{k} & =\frac{2}{\delta} \int_{0}^{\delta} f(t) \cos \left(\frac{2 \pi k t}{\delta}\right) \mathrm{d} t \\
b_{k} & =\frac{2}{\delta} \int_{0}^{\delta} f(t) \sin \left(\frac{2 \pi k t}{\delta}\right) \mathrm{d} t
\end{aligned}
$$

and

$a_{0}=\frac{2}{\delta} \int_{0}^{\delta} f(t) \mathrm{d} t$

The function $f(t)$ has infinite frequencies of the following form

$\omega=\left(\frac{2 \pi k}{\delta}\right), k \in\{1,2,3, \ldots, \infty\}$

Under the stationary boundary conditions, the values of $a_{k}$ and $a_{k 0}$ vanish for the three trajectories of interest, i.e., MJT, MST and MCT. The coefficient $b_{k}$ varies according to the trajectory as follows

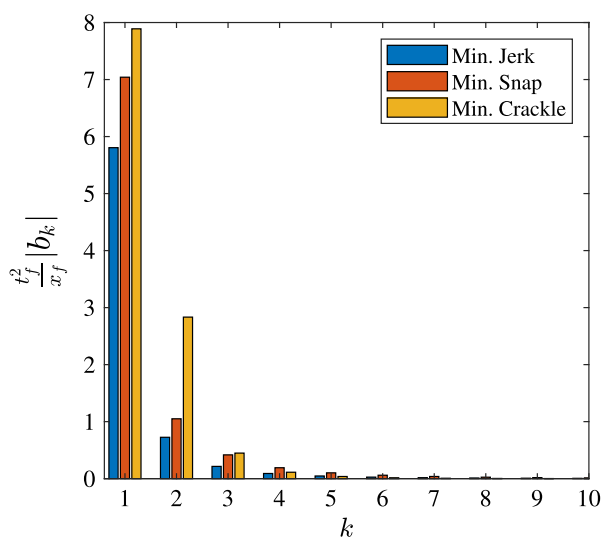

Fig. 6 The variation of the coefficient $b_{k}$ at each iteration $k$ for MJT, MST and MCT

$$
\begin{aligned}
& b_{k_{j}}=\frac{180}{\pi^{3} k^{3}} \frac{x_{f}}{t_{f}^{2}} \\
& b_{k_{s}}=-\frac{420\left(\pi^{2} k^{2}-15\right)}{\pi^{5} k^{5}} \frac{x_{f}}{t_{f}^{2}} \\
& b_{k_{c}}=-\frac{18900\left(2 \pi^{2} k^{2}-21\right)}{\pi^{7} k^{7}} \frac{x_{f}}{t_{f}^{2}}
\end{aligned}
$$

where the subscripts $j, s$ and $c$ refer to the MJT, MST and MCT, respectively.

It is worth recalling that the coefficients $b_{k}$ are the amplitudes of the sines of the function $f(t)$. In other words, the coefficients $b_{k}$ are the magnitudes of the frequencies of the signals that create the function $f(t)$. The analysis of Eq. (29) shows that $\left|b_{k_{s}}\right|>\left|b_{k_{j}}\right|$ for all $k \geq 1$. It also shows that $\left|b_{k_{c}}\right|>\left|b_{k_{j}}\right|$ for $k<5$. Figure 6 is the graphical representation of Eq. (29), and it shows that the largest magnitudes are associated with the first few frequencies, i.e., $k<5$, which means that the function $f(t)$, or simply $f$, can be well-approximated by the first four terms of its Fourier series, $f^{1 \rightarrow 4}$.

To be more rigorous, Fig. 7 shows the exact acceleration trajectory, $\ddot{x}$, which is the function $f$ in our case, along with its first four Fourier series terms, $\ddot{x}^{1 \rightarrow 4}$, and the remainder of the Fourier series terms, $\ddot{x}^{5 \rightarrow 100}$. Figure 7 also shows the percentage of the error, $\% E$, between the exact acceleration trajectory, $\ddot{x}$, and its first four-term approximation, $\ddot{x}^{1 \rightarrow 4}$. This percentage is calculated according to the following relation 


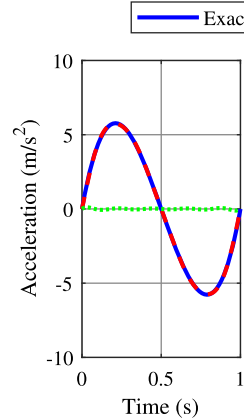

(a: MJT)

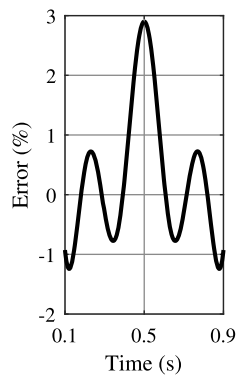

(d: MJT)

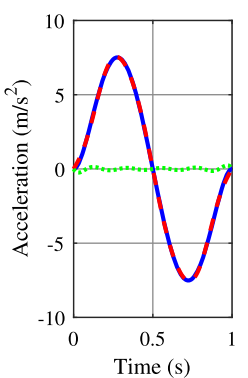

(b: MST)

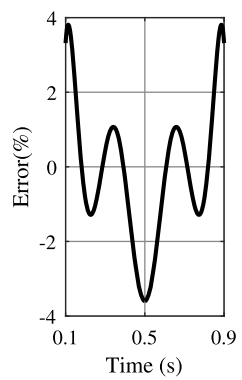

(e: MST)

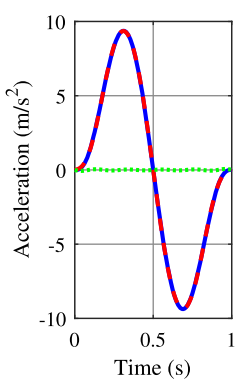

(c: MCT)

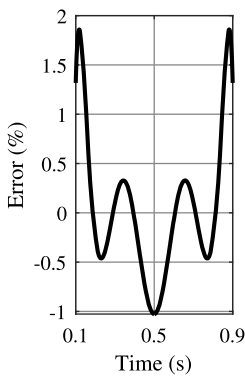

(f: MCT)

Fig. 7 The efficiency of the first four-term approximation of the acceleration trajectory for the MJT, MST and MCT

$\% E=\frac{\ddot{x}-\ddot{x}^{1 \rightarrow 4}(t)}{\ddot{x}} \times 100$

For the sake of showing a numerical example, $t_{f}$ and $x_{f}$ were chosen, arbitrarily, to be equal to one in Fig. 7. However, similar behavior is found under any other arbitrary values. Figure $7 \mathrm{a}, \mathrm{d}$ is for the MJT, Fig. 7b, e are for the MST, and lastly Fig. 7c, f, are for the MCT. It is obvious that due to the stationary boundary conditions, Eq. (30) has infinite or undefined value at the beginning and the end of the trajectory since $\ddot{x}_{0}=\ddot{x}_{f}=0$ and $f^{1 \rightarrow 4}$ can be equal to zero or very small value due to the approximation. Consequently, the range of the x-axis in the error graphs, i.e., Fig. $7 \mathrm{~d}-\mathrm{f}$, is modified to start at $t_{0}=0.1 \mathrm{~s}$, and ends at $t_{f}=0.9 \mathrm{~s}$ to avoid the infinite or undefined values at the beginning and the end of the motion. Obviously, $\ddot{x}^{1 \rightarrow 4}$ is a good approximation of $\ddot{x}$. The main conclusion from Eqs. (24)-(30) and Figs. 6 and 7 is that the MJT results in the lowest vibration excitation because it has the lowest amplitude $b_{k}$. In the given example in Fig. 7, the amplitudes are about 5.74, 7.54 and 9.35 $\mathrm{m} / \mathrm{s}^{2}$ for the MJT, MST and MCT, respectively.

4.3 The effect of the polynomial trajectory on the suspended payload vibration

The ratio between the frequency of the polynomial trajectory, $\omega$, and the natural frequency of the transportation system, $\omega_{n}$, can be found from Eqs. (21) and (28) as follows

$r=\frac{\omega}{\omega_{n}}=\frac{2 \pi k}{\delta} \sqrt{\frac{l}{g}}$

and it is always better for $r$ to be far from 1 to avoid the resonance.

Recall that $l$ is the cable length and $\delta$ represents the trajectory flight time. From Eq. (31), the values of $l$ and $\delta$ can be chosen, almost freely, to satisfy the desired frequency ratio, $r$. However, it would be better to choose $l$ and/or $\delta$ so that they reduce the suspended payload vibration.

Recalling the base excitation description in Sect. 4.1 helps borrow the concept of the transmissibility ratio, $T R$, from the base excitation vibration models. The transmissibility ratio, $T R$, is defined as the ratio between the transmitted vibration to the suspended payload and the base excitation $[42,43]$. It is worth mentioning that the concept of the transmissibility ratio is presented, in most cases in literature, as a dimensionless scalar quantity, $T R$. This is because in most cases the concept of the transmissibility ratio is presented for single DoF systems with the same dimensions and units for both the excitation and the response, e.g., distances in meter $[42,43]$. Recall that the transportation system of interest in this paper is not a single DoF system. Additionally, the excitation and the response have different dimensions and units, i.e., $\ddot{\mathbf{q}}_{\mathbf{Q}_{1,2}}$ in $\mathrm{m} / \mathrm{s}^{2}$ is the excitation and $\boldsymbol{\eta}$ in rad is the response. Consequently, the transmissibility ratio is extended in this paper to consider multiple DoFs systems; therefore, it will be a matrix, TR, with units of $\mathrm{rad} \cdot \mathrm{s}^{2} / \mathrm{m}$. The extended transmissibility ratio matrix, $\mathbf{T R}$, is derived by extending the procedure in [42]. The subscripts 1 and 2 in the definition of the matrices in Eq. (32) refer to the variables and the parameters related to the first and the second DoFs in Eq. (19), respectively.

$$
\begin{aligned}
\mathbf{T R} & =\mathbf{B}_{\mathbf{k}}{ }^{-1} \boldsymbol{\eta}_{\mathbf{A}} \\
& =\mathbf{K}_{\mathbf{s}}{ }^{-1}\left(\left[\left(\mathbf{I}-\mathbf{R}^{2}\right)^{2}+(2 \zeta \mathbf{R})^{2}\right]^{-1}\right)^{0.5}
\end{aligned}
$$

where $\mathbf{T R}=\operatorname{diag}\left(T R_{1}, T R_{2}\right) \in \mathbb{R}^{2 \times 2}$ is the transmissibility matrix, $\mathbf{B}_{\mathbf{K}}=\operatorname{diag}\left(b_{k_{1}}, b_{k_{2}}\right) \in \mathbb{R}^{2 \times 2}$ is 


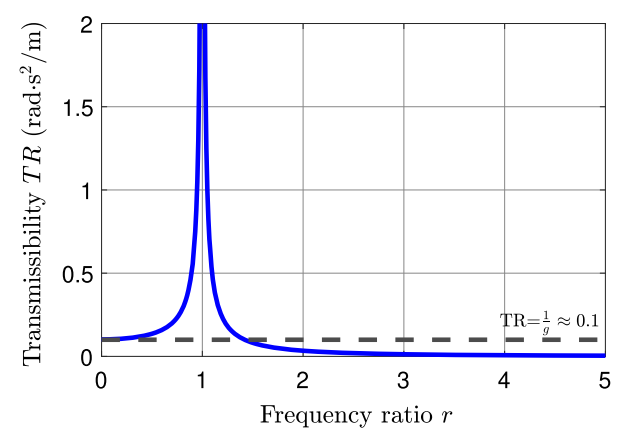

Fig. 8 Transmissibility ratio of the quadrotor with a cable suspended payload

the acceleration trajectory amplitude matrix, and $\boldsymbol{\eta}_{\mathbf{A}}=$ $\operatorname{diag}\left(\alpha_{A}, \beta_{A}\right) \in \mathbb{R}^{2 \times 2}$ is the cable angle amplitude matrix. In addition, $\mathbf{I} \in \mathbb{R}^{2 \times 2}$ is the identity matrix, $\mathbf{R}=\operatorname{diag}\left(r_{1}, r_{2}\right) \in \mathbb{R}^{2 \times 2}$ is the frequency ratio matrix, and $\zeta=\operatorname{diag}\left(\zeta_{1}, \zeta_{2}\right) \in \mathbb{R}^{2 \times 2}$ is the damping ratio matrix in case viscous damping exists in Eq. (19).

Although it is possible to have a dimensionless form of the transmissibility ratio, the authors found that the form in Eq. (32) is more suitable for the considered application because it relates the amplitudes of the quadrotor's trajectory to the cable angles straightforwardly. Considering the transportation system of interest, where there is no viscous damping, Fig. 8 is the graphical representation of Eq. (32), which can be used to specify the frequency ratio required to achieve a desired transmissibility ratio. It shows that if the system is close to the resonance, i.e., $0.95 \leq r \leq 1.05$, the transmissibility ratio, $T R$, will be more than 1 , which means that the transmitted vibration is more than the excitation. However, it is guaranteed to get $T R<\frac{1}{g}$, which is $T R<0.1 \mathrm{rad} \cdot \mathrm{s}^{2} / \mathrm{m}$, if $r>1.421$, which means $r>\sqrt{2}$. In two DoFs, this can be expressed as the transmissibility matrix is guaranteed to satisfy $\mathbf{T R}<0.1 \mathrm{I} \mathrm{rad} \cdot \mathrm{s}^{2} / \mathrm{m}$, if

$\mathbf{R}>\operatorname{diag}(\sqrt{2}, \sqrt{2})$.

From Eqs. (31), (32), and (33), two conditions can be helpful to choose $l$ and $\delta$ to reduce the transmitted vibration from the base, i.e., the quadrotor, to the suspended payload as follows

$$
\begin{aligned}
& \frac{\sqrt{l_{1}}}{\delta_{1}}>\frac{\sqrt{g}}{2 \pi k} r_{1} \\
& \frac{\sqrt{l_{2}}}{\delta_{2}}>\frac{\sqrt{g}}{2 \pi k} r_{2}
\end{aligned}
$$

where $l_{1}$ and $l_{2}$ are the required cable length to satisfy the desired transmissibility in the first and the second DoFs, respectively, $\delta_{1}$ and $\delta_{2}$ are the required trajectory flight time to satisfy the desired transmissibility in the first and the second DoFs, respectively, and $r_{1}$ and $r_{2}$ are the frequency ratios corresponding to the desired transmissibility ratios.

There are a few points, which need to be emphasized before proceeding with the discussion. First, mathematically, any combination of $l$ and $\delta$ that satisfies Eq. (34) is acceptable. Second, practically, it may not be allowed to freely choose both $l$ and $\delta$. For example, the transportation system may be constrained with a certain cable length $l$ or certain range of $l$, which means that it is not allowed to choose $l$ freely. On the other hand, the transportation task may be required to be completed in a certain amount of time, which means that $\delta$ is constrained and cannot be chosen freely. Consequently, it is important to investigate the cases, in which, only one of the two parameters, $l$ and $\delta$, can be changed freely. Third, for a certain transportation system, there is only one cable with a certain cable length, so $l_{1}$ and $l_{2}$ must be equal. Consequently, there is a need to find a way to choose if Eq. (34) results in different values of $l_{1}$ and $l_{2}$. The same concept is applied to the flight times $\delta_{1}$ and $\delta_{2}$. Fourth, the trajectory is represented as an infinite sum of linear combinations of sines and cosines based on Eq. (24), so changing $k$ in Eq. (34) has a great effect on the way we choose $l$ and $\delta$, since their values will change at each time $k$ changes.

To tackle these points, first, a clear method to identify how to address the effect of different values of $k$ on specifying $l$ and $\delta$ needs to be proposed. Second, this should be done within the assumption that only one parameter can be changed; either $l$ or $\delta$. Third, in case of two different values of $l_{1}$ and $l_{2}$, or $\delta_{1}$ and $\delta_{2}$, it is important to propose a method to choose which value should be used. The key point here is to rearrange Eq. (34) as follows

$$
\begin{aligned}
& l_{1}>\frac{w_{1}^{2}}{k^{2}} \\
& l_{2}>\frac{w_{2}^{2}}{k^{2}}
\end{aligned}
$$

and

$$
\begin{aligned}
& \delta_{1}<v_{1} k \\
& \delta_{2}<v_{2} k
\end{aligned}
$$


where, $w_{1}=\frac{r_{1} \delta_{1}}{2 \pi} \sqrt{g}, w_{2}=\frac{r_{2} \delta_{2}}{2 \pi} \sqrt{g}, v_{1}=\frac{2 \pi}{r_{1}} \sqrt{\frac{l_{1}}{g}}$, and $v_{2}=\frac{2 \pi}{r_{2}} \sqrt{\frac{l_{2}}{g}}$ are all constants.

Therefore, in Eq. (35) the flight time and frequency ratio are constrained, i.e., $\delta$ and $r$ are given. On the other hand, in Eq. (36) the cable length and frequency ratio are constrained, i.e., $l$ and $r$ are given. From Eq. (35), it is obvious that specifying $l$ using $k=1$ guarantees that this $l$ satisfies the transmissibility requirements for all $k$. Similarly, from Eq. (36), the value of $\delta$ at $k=1$ guarantees that this $\delta$ satisfies the transmissibility requirements for all $k$. If it happened that $l_{1} \neq l_{2}$, the largest of them should be chosen as it satisfies the transmissibility requirements for both DoFs. Similarly, if it happened that $\delta_{1} \neq \delta_{2}$, the smallest of them should be chosen. It is worth noting that based on Eq. (29), decreasing $\delta$ leads to increasing the amplitude, $b_{k}$, which leads to more vibration. So, it is important to emphasize the difference between the effect of the amplitude, $b_{k}$, and the transmissibility ratio, $\mathbf{T R}$. The amplitude, $b_{k}$, is the amplitude of the sinusoidal wave itself. This means that increasing this amplitude will increase the base vibration, i.e., the vibration of the quadrotor's trajectory. The transmissibility, TR, however, indicates how much of the base vibration will transfer to the suspended payload, regardless of the amount of the base vibration itself. It is also important to notice that $\delta$, in Eq. (29), is squared, while it is just $\delta$ in Eq. (31). This means that decreasing $\delta$ is not a suggested method to reduce the vibration since the effect of increasing the amplitude, $b_{k}$, will be more than the effect of decreasing the transmissibility, TR, which will lead, in most cases, to more payload vibrations.

At the end of this discussion, it is important to sum up some points. First, the transportation task can be designed so that it can satisfy the vibration level requirements. To achieve that, a clear and straightforward design method was proposed and explained. The flowchart in Fig. 9 summarizes this transportation task design process. Second, if the trajectory flight time, $\delta$, is not specified as a requirement, Eq. (29) can be used to determine $\delta$ that can achieve certain amplitude $b_{k}$. Third, it is possible to achieve a two-stage vibration reduction. The first stage is achieved by choosing the MJT as a desired trajectory. The second stage can be achieved, if possible, by decreasing the transmissibility ratio. The best way to decrease the transmissibility ratio is by changing the cable length, $l$, not the trajectory flight time $\delta$. Fourth, although the analysis in Sect. 4.2

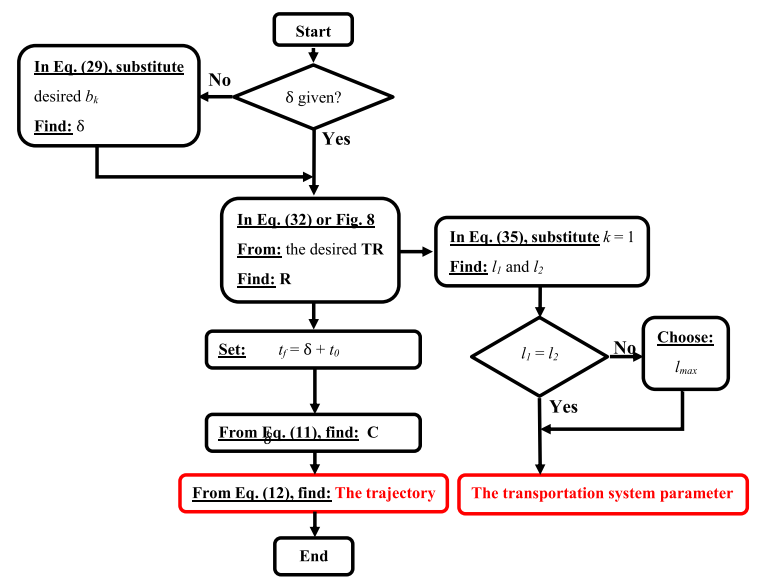

Fig. 9 The transportation task design process

have been conducted for a $1 \mathrm{D}$ trajectory, the results are still applicable to any 3D trajectory, which has a constant vertical acceleration $\ddot{z}_{Q}$. This is because the analysis in Sect. 4.1 showed that the system has a unit modal matrix, which means that the vibratory dynamics model in Eq. (19) is decoupled. For the sake of accuracy, it is worth mentioning that the transmissibility ratio calculations depend on the linearized dynamics model in Eq. (19), so if the cable angles are large enough and the dynamics goes into the nonlinear range, the transmissibility calculations will not be as accurate as it is when the cable angles are small.

\section{Simulation results}

This section presents and discusses the simulation results to illustrate two main objectives: The effectiveness of the MJT in reducing the suspended payload vibration compared to other polynomial trajectories and the use of the transmissibility ratio to provide a second stage of vibration reduction of the polynomial trajectories. Two trajectories were considered in this study: a two-point planar trajectory and a multisegment five-point 3D trajectory with sharp corners.

For the two-point trajectory, the quadrotor has to move from an initial position $\mathbf{q}_{\mathbf{Q}_{0}}=[0,0,1.2]$ to a final position $\mathbf{q}_{\mathbf{f}}=[2,2,1.2]$. The trajectory flight time is chosen to be $\delta=4$ seconds. Stationary boundary conditions are considered. This results in a four-term approximation of the amplitudes, according to Eq. (29), $b_{j}=1.2086 \mathrm{~m} / \mathrm{s}^{2}, b_{s}=1.5379 \mathrm{~m} / \mathrm{s}^{2}$ and $b_{c}=1.9947$ 
Table 1 Transportation system parameters

\begin{tabular}{llll}
\hline Parameter & Symbol & Value & Unit \\
\hline Quadrotor Parameters & & & \\
Quadrotor mass & $m_{Q}$ & 1.121 & $\mathrm{~kg}$ \\
Mass moment of inertia about $X_{B}$ & $I_{x}$ & 0.01 & $\mathrm{~kg} \cdot \mathrm{m}^{2}$ \\
Mass moment of inertia about $Y_{B}$ & $I_{y}$ & 0.0082 & $\mathrm{~kg} \cdot \mathrm{m}^{2}$ \\
Mass moment of inertia about $Z_{B}$ & $I_{z}$ & 0.0148 & $\mathrm{~kg} \cdot \mathrm{m}^{2}$ \\
Suspended Payload Parameters & & 0.2 & $\mathrm{~kg}$ \\
Suspended payload mass & $m_{L}$ & & \\
\hline
\end{tabular}

$\mathrm{m} / \mathrm{s}^{2}$, where $b_{(.)}=\sum_{k=1}^{4} b_{(.)}$and the subscripts $j, s$ and $c$ are for the MJT, MST and MCT, respectively.

For the five-point trajectory, the quadrotor is required to pass through the following five waypoints in this order: $\mathbf{q}_{\mathbf{Q}_{0}}=[0,0,1.1], \mathbf{q}_{\mathbf{Q}_{1}}=[2,0,1.25], \mathbf{q}_{\mathbf{Q}_{2}}=$ $[2,2,1.4], \mathbf{q}_{\mathbf{Q}}=[0,2,1.55]$, and $\mathbf{q}_{\mathbf{Q}}=[0,0,1.7]$. All of the four trajectory segments are required to have stationary boundary conditions in the horizontal inertial plane, i.e., $X_{I}-Y_{I}$. For the vertical motion in $Z_{I}$ direction, a constant velocity $\dot{\mathbf{z}}_{\mathbf{Q}}=[0,0,0.0429]$ $\mathrm{m} / \mathrm{s}$ is utilized. The flight time of each of the four trajectory segments is 3.6 seconds. Using Eq. (29), the four-term approximation of the amplitudes can be found as $b_{j}=1.1162 \mathrm{~m} / \mathrm{s}^{2}, b_{s}=1.4204 \mathrm{~m} / \mathrm{s}^{2}$ and $b_{c}=1.8423 \mathrm{~m} / \mathrm{s}^{2}$. This multi-segment 3D fivepoint trajectory was chosen on purpose because the sharp corners and the stationary boundary conditions of all trajectory segments in the horizontal inertial plane will break the smoothness between the trajectory segments and increase the payload vibration. This will help demonstrate the effectiveness of the proposed methods to reduce the suspended payload vibration.

The suspended payload vibration is quantified by the two cable angles, $\alpha$ and $\beta$. The trajectory that has the lowest maximum cable angles, $\alpha$ and $\beta$, is said to be the trajectory that results in the lowest payload vibration. The transportation system parameters are indicated in Table 1.

\subsection{The MJT and the suspended payload vibration}

In this subsection, the effect of the polynomial trajectory degree on the suspended payload vibration is investigated. The cable length is chosen, arbitrarily, to be $l=1 \mathrm{~m}$. The MJT, the MST and the MCT are considered. Equation (31) is used to find the frequency ratio matrix, which is used in Eq. (32) and Fig. 8 to find the transmissibility ratio matrix. The values of the diagonal elements of these two diagonal matrices at cable length $l=1 \mathrm{~m}$ are shown in Table 2. First, the simulation results of the two-point planer trajectory are presented and then the results of the multi-segment fivepoint 3D trajectory. The main objective of the results in this subsection is to show that the MJT results in the lowest payload vibration compared to the rest of the polynomial trajectories.

\subsubsection{Two-point planar trajectory}

Although the focus of this paper is to investigate the effect of the polynomial trajectories on the suspended payload vibration, it is a good idea to give a sense of how the polynomial trajectories' vibration behavior is compared to other common trajectories. Consequently, the simulation results of the step trajectory are also considered to give the reader an idea about how good the performance of the polynomial trajectory is. The path of the quadrotor and the suspended payload are presented in Fig. 10, while the cable angles, $\alpha$ and $\beta$, are shown in Fig. 11.

These results show, obviously, that the MJT has the lowest suspended payload vibration, in both simulation and experimental results, among the rest of the polynomial trajectories.

Using the four-term approximation values in Sect. 5 and the transmissibility ratio matrix presented in Table 2, the maximum cable angles can be calculated. A summary of the maximum calculated and simulated cable angles is presented in Table 3 . The results in Table 3 show that the calculated results are very close to the simulation results. 
Table 2 The diagonal elements of the frequency and transmissibility ratios matrices for different cable lengths for both two-point and five-point trajectories

\begin{tabular}{|c|c|c|c|c|c|c|}
\hline \multirow[t]{2}{*}{ Trajectory } & \multicolumn{2}{|l|}{$l=0.5 \mathrm{~m}$} & \multicolumn{2}{|l|}{$l=1 \mathrm{~m}$} & \multicolumn{2}{|l|}{$l=16 \mathrm{~m}$} \\
\hline & $\mathbf{R}$ & $\mathbf{T R}\left(\mathrm{rad} \cdot \mathrm{s}^{2} / \mathrm{m}\right)$ & $\mathbf{R}$ & $\mathbf{T R}\left(\mathrm{rad} \cdot \mathrm{s}^{2} / \mathrm{m}\right)$ & $\mathbf{R}$ & $\mathbf{T R}\left(\mathrm{rad} \cdot \mathrm{s}^{2} / \mathrm{m}\right)$ \\
\hline Two-point & $(0.3546,0.3546)$ & $(0.1166,0.1166)$ & $(0.5015,0.5015)$ & $(0.1362,0.1362)$ & $(2,2)$ & $(0.034,0.034)$ \\
\hline Five-point & $(0.4053,0.4053)$ & $(0.122,0.122)$ & $(0.5732,0.5732)$ & $(0.1518,0.1518)$ & $(2.3,2.3)$ & $(0.024,0.024)$ \\
\hline
\end{tabular}

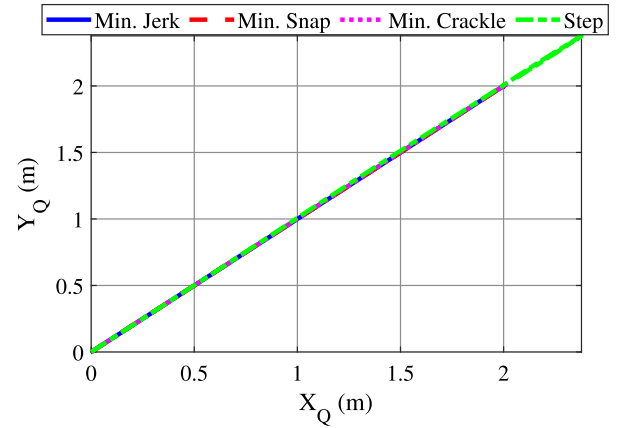

(a) Quadrotor path

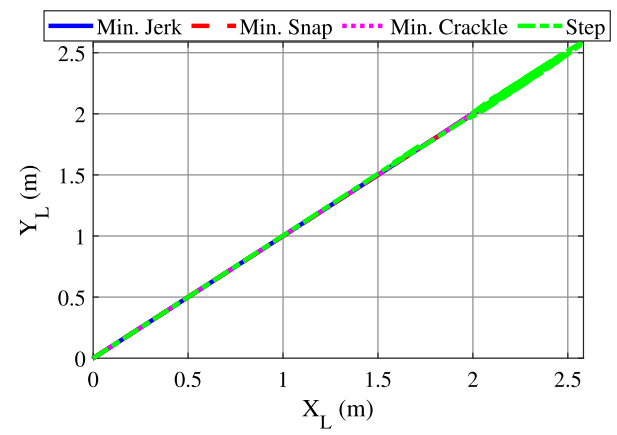

(b) Suspended payload path

Fig. 10 The simulation of the paths of the quadrotor and the suspended payload (two-point trajectory, $l=1 \mathrm{~m}$ )

To sum up, the results of the two-point planar trajectory show that the MJT results in the lowest payload vibration among the rest of the polynomial trajectories.

\subsubsection{Five-point $3 D$ trajectory}

The 3D paths of the quadrotor and the suspended payload are shown in Fig. 12. The cable angles, $\alpha$ and $\beta$, are shown in Fig. 13. From these results, the MJT, obviously, has the lowest cable angle among the rest of the polynomial trajectories.

Similar to the two-points trajectory in Sect. 5.1.1, the maximum cable angles are calculated and a sum-
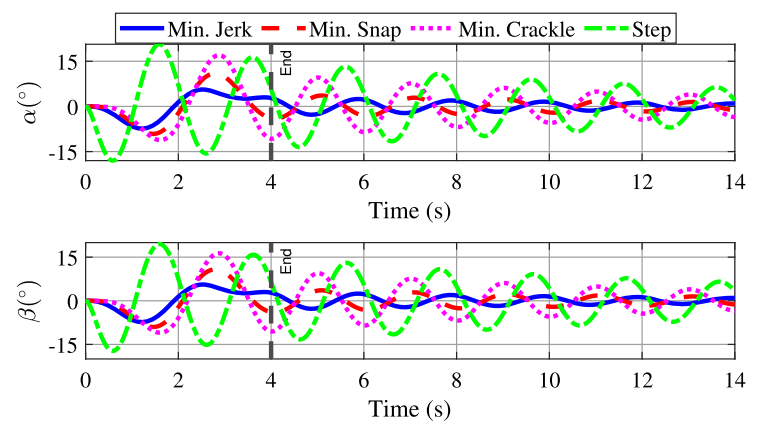

Fig. 11 The simulation results of the cable angles $\alpha$ and $\beta$ (twopoint trajectory, $l=1 \mathrm{~m}$ )

mary of the maximum calculated and simulated cable angles is presented in Table 3. Except for the MJT, there is a difference between the calculated angles and the simulation results. The main reason for this difference, which does not appear in the two-point trajectory case, is that the calculated angles were calculated for each trajectory segment separately. This means assuming zero initial condition at each way point, i.e., zero cable angles and zero cable angles rates. Contrarily, in the actual case, each trajectory segment starts with a nonzero initial conditions, which are equal to the actual final conditions of the previous segment because the suspended payload reaches the intermediate waypoints with oscillations, i.e., nonzero cable angles and angle rates. Figure 14 shows the cable angles and the cable angles' rates at each of the intermediate waypoints, i.e., $t=\{3.5,7,10.5\}$ second, which are indicated by the vertical dashed lines. Figure 14 also explains why the MJT results are close to the calculated results. The reason is that the MJT reaches the intermediate waypoints with cable angles and cable angles' rates close to zero.

In conclusion, the results of the five-point 3D trajectory show that the MJT results in the lowest payload vibration compared to the rest of the polynomial trajectories. 
Table 3 Maximum cable angles $(l=1 \mathrm{~m})$

\begin{tabular}{|c|c|c|c|c|c|c|}
\hline & \multicolumn{2}{|c|}{ Calculated } & \multicolumn{2}{|c|}{ Simulation } & \multicolumn{2}{|c|}{ Experimental } \\
\hline & $\alpha_{\max }$ & $\beta_{\max }$ & $\alpha_{\max }$ & $\beta_{\max }$ & $\alpha_{\max }$ & $\beta_{\max }$ \\
\hline \multicolumn{7}{|c|}{ Two-point Trajectory } \\
\hline MJT & $9.4^{\circ}$ & $9.4^{\circ}$ & $7.3^{\circ}$ & $7.3^{\circ}$ & $14.1^{\circ}$ & $12.7^{\circ}$ \\
\hline MST & $12^{\circ}$ & $12^{\circ}$ & $10.7^{\circ}$ & $10.7^{\circ}$ & $19^{\circ}$ & $17.5^{\circ}$ \\
\hline $\mathrm{MCT}$ & $15.6^{\circ}$ & $15.6^{\circ}$ & $17^{\circ}$ & $16.3^{\circ}$ & $32^{\circ}$ & $32.7^{\circ}$ \\
\hline \multicolumn{7}{|c|}{ Five-point Trajectory } \\
\hline MJT & $9.7^{\circ}$ & $9.7^{\circ}$ & $9.9^{\circ}$ & $9.9^{\circ}$ & $21.5^{\circ}$ & $23.2^{\circ}$ \\
\hline MST & $12.3^{\circ}$ & $12.3^{\circ}$ & $23^{\circ}$ & $23.2^{\circ}$ & $30.2^{\circ}$ & $32.1^{\circ}$ \\
\hline MCT & $16^{\circ}$ & $16^{\circ}$ & $34^{\circ}$ & $34.8^{\circ}$ & $43^{\circ}$ & $41.5^{\circ}$ \\
\hline
\end{tabular}

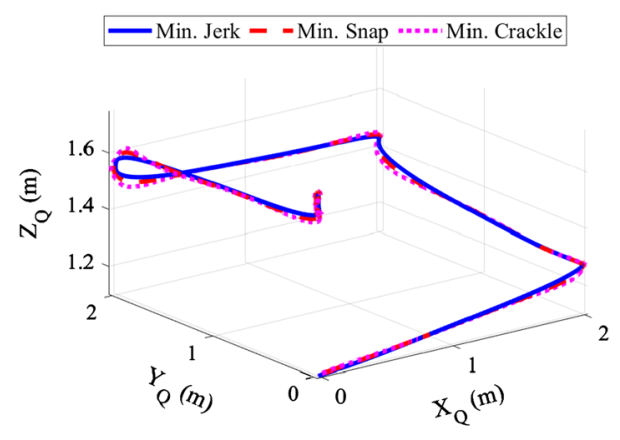

(a) Quadrotor 3D path

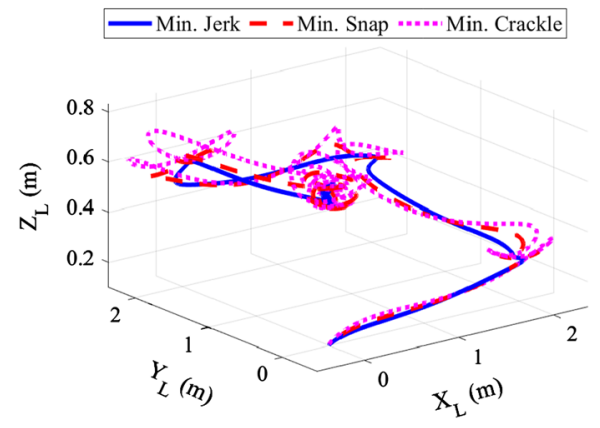

(b) Suspended payload 3D path

Fig. 12 The simulation of the 3D paths of the quadrotor and the suspended payload (five-point trajectory, $l=1 \mathrm{~m}$ )

\subsection{The transmissibility and the suspended payload vibration}

In this subsection, the effect of the transmissibility ratio on the payload vibration is investigated. After reducing the payload vibration by designing a MJT, the transmissibility ratio can be used to provide a second stage of vibration reduction. In our case, Fig. 8 and Eq. (32)
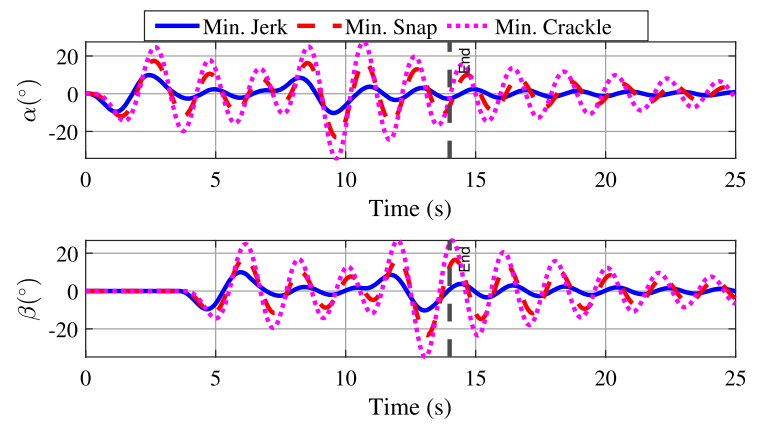

Fig. 13 The simulation results of the cable angles $\alpha$ and $\beta$ (fivepoint trajectory, $l=1 \mathrm{~m}$ )

tell that it is possible to reduce the suspended payload vibration level by reducing the transmissibility ratio, TR, through two different methods. The first method is by reducing the frequency ratio, $\mathbf{R}$, to be less than $\operatorname{diag}(0.5015,0.5015)$ in case of the two-point trajectory, and less than $\operatorname{diag}(0.5732,0.5732)$ in case of the five-point trajectory. Alternatively, the payload vibration level can be reduced by increasing the frequency ratio, $\mathbf{R}$, to be more than $\operatorname{diag}(1.3223,1.3223)$ in case of the two-point trajectory, and more than $\operatorname{diag}(1.2929,1.2929)$ in case of the five-point trajectory. In this paper, we would like to demonstrate the case where $\mathbf{T R}<0.1 \mathrm{I} \mathrm{rad} \cdot \mathrm{s}^{2} / \mathrm{m}$, so $\mathbf{R}>$ $\operatorname{diag}(\sqrt{2}, \sqrt{2})$ is considered for both trajectories. As discussed in Sect. 4.3, the selected method to achieve that is to change the cable length. Consequently, there are two different ways to provide a second stage of vibration reduction. For the two-point trajectory, these two ways are either $l<1 \mathrm{~m}$ or $l>7.95 \mathrm{~m}$, while for the five-point trajectories, the two ways are either $l<1 \mathrm{~m}$ 

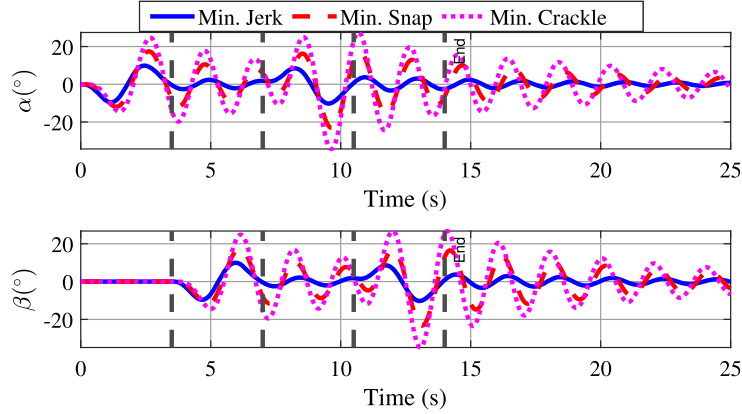

(a) Cable angles $\alpha$ and $\beta$
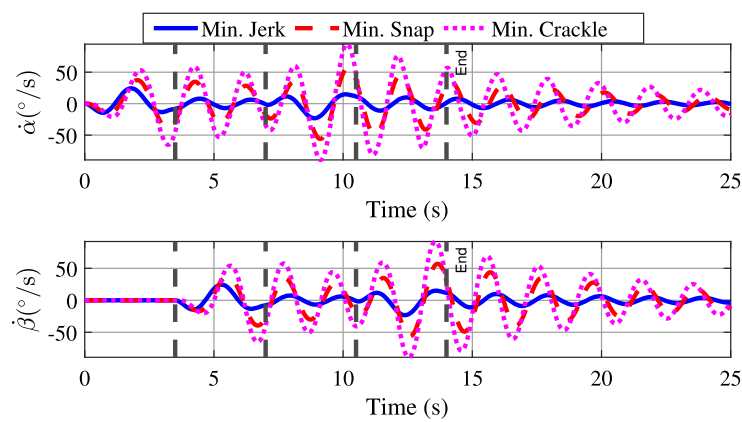

(b) Cable angles' rates $\dot{\alpha}$ and $\dot{\beta}$

Fig. 14 Initial conditions of $\alpha, \beta, \dot{\alpha}$ and $\dot{\beta}$ at the intermediate waypoints (five-point trajectory, $l=1 \mathrm{~m}$ )

or $l>6.088 \mathrm{~m}$. For consistency, a short cable of length $l=0.5 \mathrm{~m}$ and a long cable of length $l=16 \mathrm{~m}$ are chosen to investigate the effect of reducing the transmissibility on the suspended payload vibration level for both trajectories. The corresponding frequency and transmissibility ratios of these cable lengths for the two trajectories are shown in Table 2. Due to the space limitations, the experiments for the long cable case cannot be conducted, so only the simulation results are included for the long cable case.

\subsubsection{Two-point planar trajectory}

For the short cable, i.e., $l=0.5 \mathrm{~m}$, the simulation results of the two cable angles, $\alpha$ and $\beta$, are shown in Fig. 15. For the long cable, i.e., $l=16 \mathrm{~m}$, the simulation results of the cable angles, $\alpha$ and $\beta$, are shown in Fig. 16.

First, these results confirm that the MJT has the lowest payload vibration among the rest of the polynomial trajectories, which is the main conclusion in Sect. 5.1. Second, it shows that the transmissibility ratio can be used to provide a second stage of vibration reduction since All of the cable angles in Figs. 15 and 16 are less
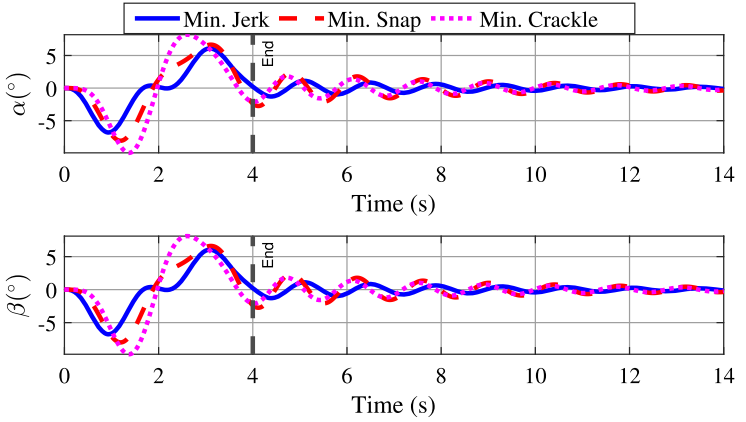

Fig. 15 The simulation results of the cable angles $\alpha$ and $\beta$ (twopoint trajectory, $l=0.5 \mathrm{~m}$ )
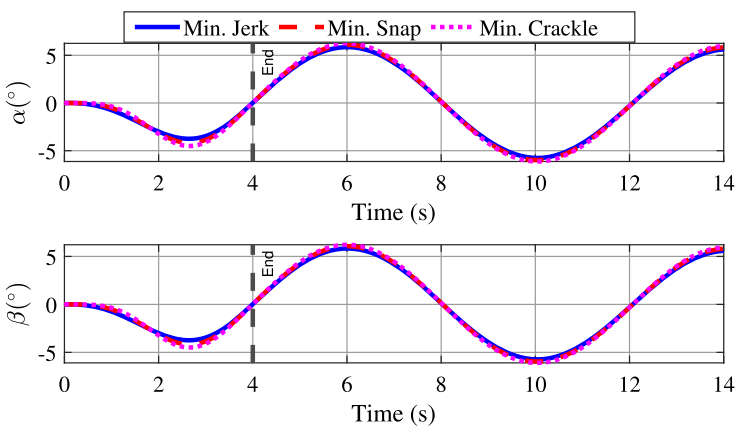

Fig. 16 The simulation results of the cable angles $\alpha$ and $\beta$ (twopoint trajectory, $l=16 \mathrm{~m}$ )

than their analogs in Fig. 11. A summary of the maximum calculated and simulated cable angles for both the short and long cables is presented in Tables 4 and 5, respectively.

The main conclusion here is that decreasing the transmissibility ratio can provide a second stage of vibration reduction for the two-points trajectory, and this can be achieved using either a short or a long cable.

\subsubsection{Five-point $3 D$ trajectory}

For the short cable, i.e., $l=0.5 \mathrm{~m}$, the simulation results of the two cable angles, $\alpha$ and $\beta$, are shown in Fig. 17. For the long cable, i.e., $l=16 \mathrm{~m}$, the simulation results of the cable angles, $\alpha$ and $\beta$, are shown in Fig. 18.

Similar to the two-point trajectory results in Sect. 5.2.1, in addition to confirming that the MJT has the lowest payload vibration among the rest of the polynomial, the results show that the transmissibility ratio can be used to provide a second stage of vibration reduction. All of the cable angles in this subsection are less than their analogs in Sect. 5.1.2. A summary of the max- 
Table 4 Maximum cable angles $(l=0.5 \mathrm{~m})$

\begin{tabular}{|c|c|c|c|c|c|c|}
\hline & \multicolumn{2}{|c|}{ Calculated } & \multicolumn{2}{|c|}{ Simulation } & \multicolumn{2}{|c|}{ Experimental } \\
\hline & $\alpha_{\max }$ & $\beta_{\max }$ & $\alpha_{\max }$ & $\beta_{\max }$ & $\alpha_{\max }$ & $\beta_{\max }$ \\
\hline \multicolumn{7}{|c|}{ Two-point Trajectory } \\
\hline MJT & $8.1^{\circ}$ & $8.1^{\circ}$ & $6.8^{\circ}$ & $6.7^{\circ}$ & $12.9^{\circ}$ & $13^{\circ}$ \\
\hline MST & $10.3^{\circ}$ & $10.3^{\circ}$ & $8^{\circ}$ & $7.9^{\circ}$ & $16^{\circ}$ & $16.5^{\circ}$ \\
\hline MCT & $13.3^{\circ}$ & $13.3^{\circ}$ & $9.9^{\circ}$ & $9.7^{\circ}$ & $17.5^{\circ}$ & $17.5^{\circ}$ \\
\hline \multicolumn{7}{|c|}{ Five-point Trajectory } \\
\hline MJT & $7.8^{\circ}$ & $7.8^{\circ}$ & $9.1^{\circ}$ & $9.2^{\circ}$ & $18.2^{\circ}$ & $18.2^{\circ}$ \\
\hline MST & $9.9^{\circ}$ & $9.9^{\circ}$ & $11^{\circ}$ & $11^{\circ}$ & $25^{\circ}$ & $22.1^{\circ}$ \\
\hline MCT & $12.9^{\circ}$ & $12.9^{\circ}$ & $14.4^{\circ}$ & $14.5^{\circ}$ & $30^{\circ}$ & $28.2^{\circ}$ \\
\hline
\end{tabular}

Table 5 Maximum cable angles $(l=16 \mathrm{~m})$

\begin{tabular}{|c|c|c|c|c|}
\hline & \multicolumn{2}{|c|}{ Calculated } & \multicolumn{2}{|c|}{ Simulation } \\
\hline & $\alpha_{\max }$ & $\beta_{\max }$ & $\alpha_{\max }$ & $\beta_{\max }$ \\
\hline \multicolumn{5}{|c|}{ Two-point trajectory } \\
\hline MJT & $2.4^{\circ}$ & $2.4^{\circ}$ & $5.8^{\circ}$ & $5.8^{\circ}$ \\
\hline MST & $3^{\circ}$ & $3^{\circ}$ & $6.1^{\circ}$ & $6^{\circ}$ \\
\hline MCT & $3.9^{\circ}$ & $3.9^{\circ}$ & $6.2^{\circ}$ & $6.2^{\circ}$ \\
\hline \multicolumn{5}{|c|}{ Five-point Trajectory } \\
\hline MJT & $1.5^{\circ}$ & $1.5^{\circ}$ & $6^{\circ}$ & $6^{\circ}$ \\
\hline MST & $2^{\circ}$ & $2^{\circ}$ & $6.2^{\circ}$ & $6.2^{\circ}$ \\
\hline $\mathrm{MCT}$ & $2.5^{\circ}$ & $2.5^{\circ}$ & $6.3^{\circ}$ & $6.3^{\circ}$ \\
\hline
\end{tabular}

imum calculated and simulated cable angles for both the short and long cables is presented in Tables 4 and 5, respectively.

The main conclusion here is that decreasing the transmissibility can provide a second stage of vibration reduction for the five-point trajectory, and this can be achieved using either a short or a long cable.

\section{Experimental validation}

In this section, the results and the findings in Sect. 5 are verified and validated experimentally. In particular, this section verifies and validates that the MJT results in the lowest payload vibration among the rest of the polynomial trajectories and the transmissibility ratio can be used to provide a second stage of vibration reduction.

The experiments are conducted using York University's Autonomous Unmanned Vehicle (YU-AUV)
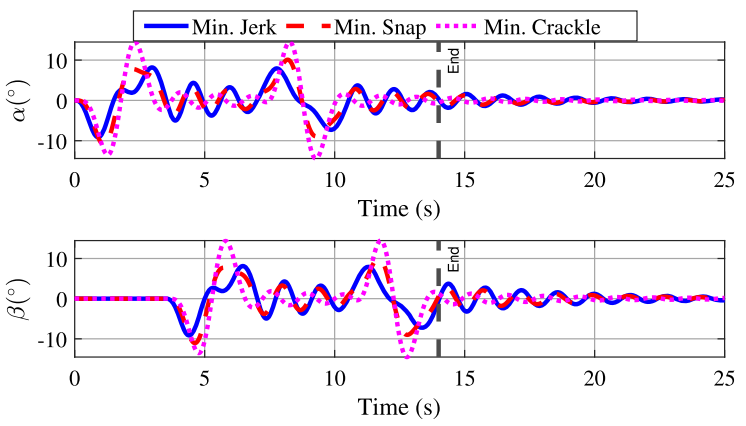

Fig. 17 The simulation results of the cable angles $\alpha$ and $\beta$ (fivepoint trajectory, $l=0.5 \mathrm{~m}$ )

facility. Figure 19 shows the experimental setup, which consists of 16 Flex 13 cameras, which form the OptiTrack motion capture system. They are connected via USB to the workstation, which is equipped with Quanser's QUARC software and MATLAB to provide the real-time control commands. The workstation is wired-connected to the router, while the router is con- 

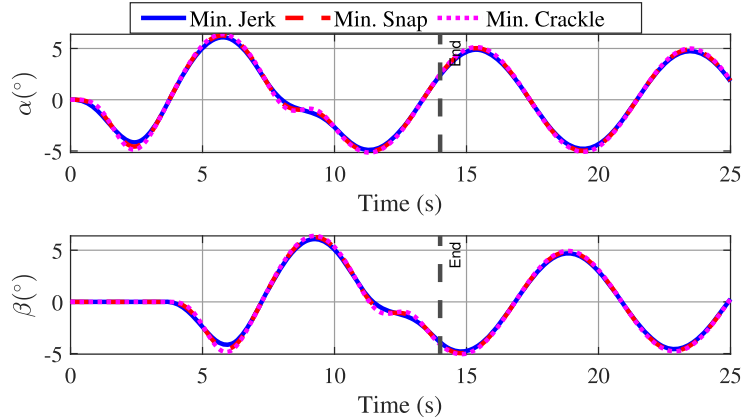

Fig. 18 The simulation results of the cable angles $\alpha$ and $\beta$ (fivepoint trajectory, $l=16 \mathrm{~m}$ )

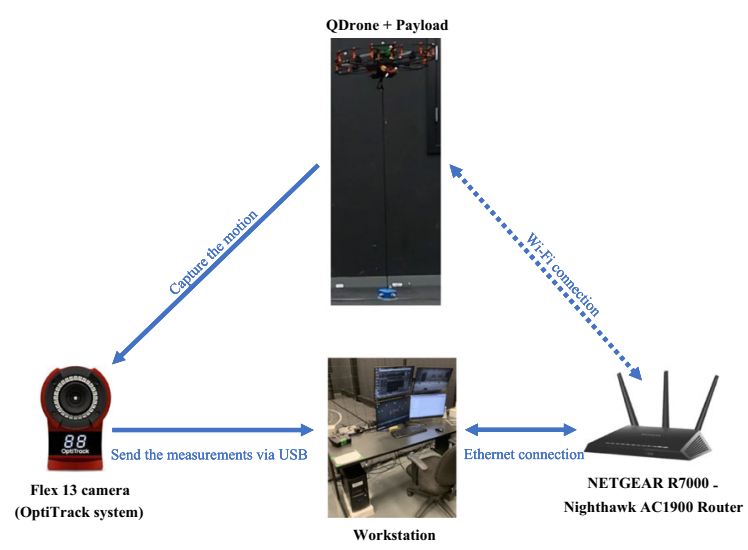

Fig. 19 Experimental setup

nected, wirelessly, to the QDrone quadrotor via WiFi. The QDrone quadrotor is equipped with the Intel Aero Compute board, which has Intel Atom x7-Z8750 quad-core 64-bit $2.56 \mathrm{GHz}$ processor. The test videos are available at https://youtu.be/6umbq97AK00.

\subsection{Validation of the effectiveness of the MJT in vibration reduction}

\subsubsection{Two-point planar trajectory}

In this subsection, a cable length $l=1 \mathrm{~m}$ is considered. The experimental results for the cable angles, $\alpha$ and $\beta$, are shown in Fig. 20. These experimental results confirm that the MJT results in the lowest payload vibration compared to the rest of the polynomial trajectories. Table 3 confirms the same conclusion with numbers; however, the experimental results are not as close to the calculated results as the simulation results. One of the main reasons is that the calculated results
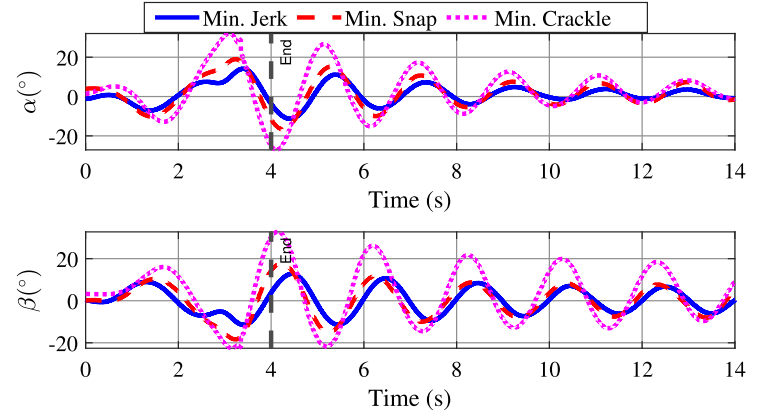

Fig. 20 The experimental results of the cable angles $\alpha$ and $\beta$ (two-point trajectory, $l=1 \mathrm{~m}$ )

are based on the linearized dynamics model, which is not the actual case, especially for large angles, e.g., the MCT. Additionally, a deeper look at the results shows that the larger angles in the experimental results are near the end of the motion, whereas in the rest of the motion the cable angles are almost similar to the calculated cable angles. This is mainly due to the inertia of the system and actual control commands. Near the end of the motion, the quadrotor has to change its attitude aggressively to stop, which leads to this larger angles near the end of the motion. This can be noticed obviously in the experimental results near 4 seconds and in the test videos at https://youtu.be/6umbq97AK00. This may be improved by improving the controller; however, this is not the objective of this paper.

The main conclusion is that it is validated experimentally that the MJT results in the lowest payload vibration among the rest of the polynomial trajectories regardless of the quality of the controller.

\subsubsection{Five-point $3 D$ trajectory}

In this subsection, a cable length $l=1 \mathrm{~m}$ is considered. The experimental results for the cable angles, $\alpha$ and $\beta$, are shown in Fig. 21. Similar to the two-point trajectory, these experimental results confirm that the MJT results in the lowest payload vibration compared to the rest of the polynomial trajectories. This can be proven also by looking at the cable angles in Table 3. From Table 3, one can notice larger difference between the calculated and the experimental results. The main reasons for this difference are mentioned in Sects. 5.1.2 and 6.1.1. Additionally, recall that this trajectory was designed on purpose with sharp corners and instantaneous stops at the intermediate waypoints of this trajectory to increase the 

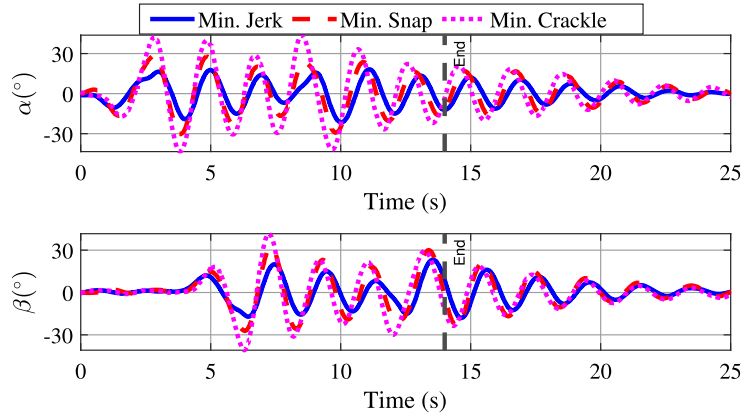

Fig. 21 The experimental results of the cable angles $\alpha$ and $\beta$ (five-point trajectory, $l=1 \mathrm{~m}$ )

vibration. These sources of excessive vibration combined with the actual inertia effect increase the vibration in the reality. This can be noticed, obviously, in the test videos at https://youtu.be/6umbq97AK00.

This subsection validates that the MJT has the lowest payload vibration compared to the rest of the polynomial trajectories.

\subsection{Validation of the transmissibility ratio effect}

\subsubsection{Two-point planar trajectory}

Recall that the experimental validation is only for the short cable, i.e., $l=0.5 \mathrm{~m}$. The experimental results for the cable angles, $\alpha$ and $\beta$, are shown in Fig. 22. The main results are summarized in Table 4 . The reasons for the difference in the experimental results are explained in Sect. 6.1.1. The results of the two-point trajectory show that the MJT has the lowest vibration level among the rest of the polynomial trajectories. More importantly, they show that the transmissibility ratio can be used to provide a second stage of vibration reduction.

\subsubsection{Five-point $3 D$ trajectory}

Here, we provide an experimental validation for the short cable case only, i.e., $l=0.5 \mathrm{~m}$. The experimental results for the cable angles, $\alpha$ and $\beta$, are shown in Fig. 23, which are summarized in Table 4. As explained in Sect. 6.1.2, there are some differences between the experimental and the calculated results. However, the results show that the MJT still has the lowest vibration level among the rest of the polynomial trajectories. More importantly, they show that the transmissibility
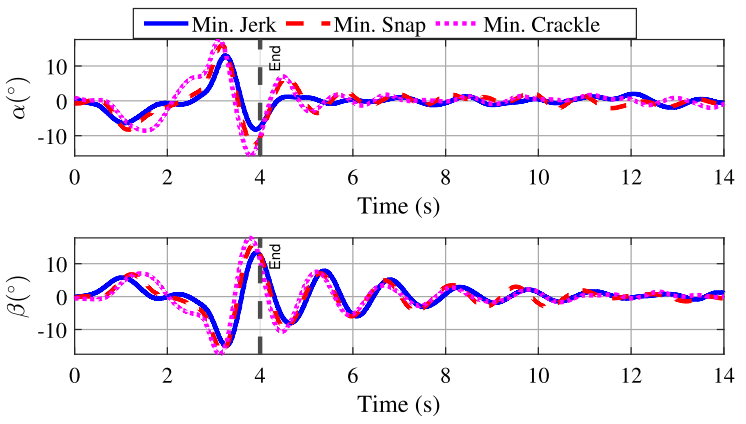

Fig. 22 The experimental results of the cable angles $\alpha$ and $\beta$ (two-point trajectory, $l=0.5 \mathrm{~m}$ )
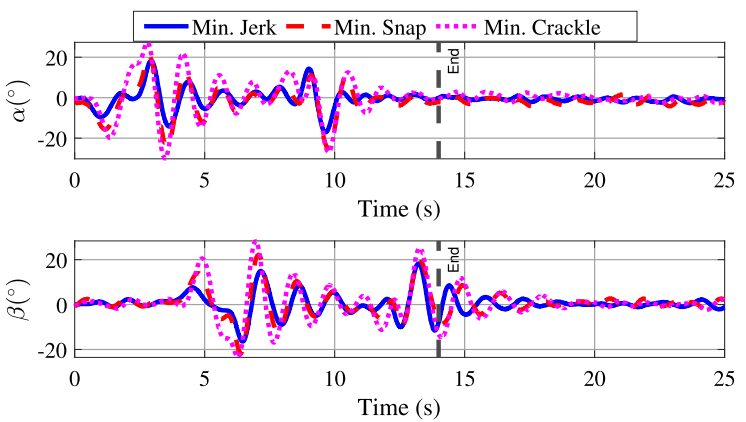

Fig. 23 The experimental results of the cable angles $\alpha$ and $\beta$ (five-point trajectory, $l=0.5 \mathrm{~m}$ )

ratio can be used to provide a second stage of vibration reduction.

\section{Conclusions}

This paper considers a transportation system consisting of a quadrotor and a cable suspended payload. It investigates the effect of the polynomial trajectories on the suspended payload vibration and provides a method to design a polynomial trajectory that can reduce the suspended payload vibration. The vibration analysis of the transportation system and the polynomial trajectories showed that the MJT results in the lowest payload vibration compared to the rest of the polynomial trajectories. A second stage of payload vibration reduction was provided by applying the concept of the transmissibility ratio to reduce the transmitted vibration from the quadrotor to the payload regardless the vibration level of the quadrotor itself. The proposed approach depends on the inherent features of the MJTs to reduce the payload vibration for any certain controller and path regardless the type of the controller and/or the geom- 
etry of the path themselves. Two different trajectories were considered in this paper: a two-point planar trajectory and a multi-segment five-point 3D trajectory with sharp corners. Both the simulation and the experimental results of these two trajectories were discussed. It was verified that the MJT has the lowest payload vibration among the rest of the polynomial trajectories, and the transmissibility ratio can be used to provide a second stage of payload vibration reduction. Investigating the effect of the offset between the center of mass of the quadrotor and the attaching point on the payload vibration is a topic for future exploration.

Acknowledgements The authors would like to thank Dr. Ti Chen for his help in running the experiments and Dr. Adeel Akhtar for his help in making the animations.

Author contributions Hassan Alkomy helped in conceptualization, methodology, writing-original draft, visualization, investigation, validation.

Jinjun Shan contributed to supervision, project administration, writing-original draft.

Funding No funds, grants, or other support was received.

\section{Declarations}

Conflict of interest The authors declare that they do not have any financial or nonfinancial conflict of interests.

\section{Appendix}

\section{A Fourier coefficient for polynomial trajectories with general boundary conditions}

The cosines coefficients, $a_{k}$, for the different polynomial trajectories can be found as follows

$$
\begin{aligned}
& a_{k_{j}}=\frac{1}{\pi^{2} k^{2} t_{f}}\left(3\left(\ddot{x}_{0}+\ddot{x}_{f}\right) t_{f}+6\left(\dot{x}_{0}-\dot{x}_{f}\right)\right) \\
& a_{k_{s}}=\frac{1}{2 \pi^{4} k^{4} t_{f}}\left(-\left(\pi^{2} k^{2}-15\right)\left(\dddot{x}_{0}-\dddot{x}_{f}\right) t_{f}^{2}\right. \\
& \left.\quad+90\left(\ddot{x}_{0}+\ddot{x}_{f}\right) t_{f}+180\left(\dot{x}_{0}-\dot{x}_{f}\right)\right) \\
& a_{k_{c}}=\frac{1}{4 \pi^{6} k^{6} t_{f}}\left(-10\left(\pi^{2} k^{2}-10.5\right)\left(\dddot{x}_{0}+\dddot{x}_{f}\right) t_{f}^{3}\right. \\
& -2\left(\pi^{4} k^{4}+45 \pi^{2} k^{2}-630\right)\left(\dddot{x}_{0}-\dddot{x}_{f}\right) t_{f}^{2} \\
& -420\left(\pi^{2} k^{2}-15\right)\left(\ddot{x}_{0}+\ddot{x}_{f}\right) t_{f} \\
& \left.-840\left(\pi^{2} k^{2}-15\right)\left(\dot{x}_{0}-\dot{x}_{f}\right)\right)
\end{aligned}
$$

The sines coefficients, $b_{k}$, for the different polynomial trajectories can be found as follows

$$
\begin{aligned}
b_{k_{j}} & =\frac{1}{\pi^{3} k^{3} t_{f}^{2}} \\
& \left(\left(\pi^{2} k^{2}-15\right)\left(\ddot{x}_{0}-\ddot{x}_{f}\right) t_{f}^{2}-90\left(\dot{x}_{0}+\ddot{x}_{f}\right) t_{f}\right. \\
& \left.-180\left(x_{0}-x_{f}\right)\right) \\
b_{k_{s}} & =\frac{1}{2 \pi^{5} k^{5} t_{f}^{2}}\left(10\left(\pi^{2} k^{2}-10.5\right)\left(\dddot{x}_{0}+\dddot{x}_{f}\right) t_{f}^{3}\right. \\
& +2\left(\pi^{4} k^{4}+45 \pi^{2} k^{2}-630\right)\left(\ddot{x}_{0}-\ddot{x}_{f}\right) t_{f}^{2} \\
& +420\left(\pi^{2} k^{2}-15\right)\left(\dot{x}_{0}+\dot{x}_{f}\right) t_{f} \\
& \left.+840\left(\pi^{2} k^{2}-15\right)\left(x_{0}-x_{f}\right)\right) \\
b_{k_{c}} & =\frac{1}{4 \pi^{7} k^{7} t_{f}^{2}} \\
& \left(-\left(\pi^{4} k^{4}-105 \pi^{2} k^{2}+945\right)\left(\dddot{x}_{0}-\dddot{x}_{f}\right) t_{f}^{4}\right. \\
& +1890\left(\pi^{2} k^{2}-10\right)\left(\dddot{x}_{0}+\dddot{x}_{f}\right) t_{f}^{3} \\
& +4\left(\pi^{6} k^{6}+4095 \pi^{2} k^{2}-42525\right)\left(\ddot{x}_{0}-\ddot{x}_{f}\right) t_{f}^{2} \\
& +75600\left(\pi^{2} k^{2}-10.5\right)\left(\dot{x}_{0}+\dot{x}_{f}\right) t_{f} \\
& \left.+151200\left(\pi^{2} k^{2}-10.5\right)\left(x_{0}-x_{f}\right)\right)
\end{aligned}
$$

The coefficients, $a_{0}$, is the same for all of the different polynomial trajectories, since it is basically the integration of the trajectory's acceleration, i.e., the velocity. The coefficient, $a_{0}$, can be found as follows

$a_{0}=\frac{-2}{t_{f}}\left(\dot{x}_{0}-\dot{x}_{f}\right)$

\section{References}

1. Fink, J., Michael, N., Kim, S., Kumar, V.: Planning and Control for Cooperative Manipulation and Transportation with Aerial Robots. The International Journal of Robotics Research 30(3), 324-334 (2010)

2. Loianno, G., Kumar, V.: Cooperative Transportation Using Small Quadrotors Using Monocular Vision and Inertial Sensing. IEEE Robotics and Automation Letters 3(2), 680687 (2018)

3. Rastgoftar, H., Atkins, E.M.: "Continuum Deformation of a Multiple Quadcopter Payload Delivery Team without InterAgent Communication," in 2018 International Conference on Unmanned Aircraft Systems (ICUAS), Dallas, TX, USA, pp. 539-548 (2018)

4. Chen, T., Shan, J.: "Cooperative Transportation of CableSuspended Slender Payload Using Two Quadrotors," in 2019 IEEE International Conference on Unmanned Systems (ICUS), Beijing, China, pp. 432-437 (2019) 
5. Cardona, G.A., Tellez-Castro, D., Mojica-Nava, E.: Cooperative Transportation of a Cable-Suspended Load by Multiple Quadrotors. IFAC-Papers On Line 52(20), 145-150 (2019)

6. Chen, T., Shan, J.: A Novel Cable-Suspended Quadrotor Transportation System: From Theory to Experiment. Aerospace Science and Technology 104, 105974 (2020)

7. Ding, X., Guo, P., Xu, K., Yu, Y.: A Review of Aerial Manipulation of Small-Scale Rotorcraft Unmanned Robotic Systems. Chinese Journal of Aeronautics 32 (1), 200-214 (2019)

8. Villa, D.K.D., Brandão, A.S., Sarcinelli-Filho, M.: "A Survey on Load Transportation Using Multirotor UAVs," Journal of Intelligent \& Robotic Systems, vol. 98, no. 2, pp. 267296 (2020) [Online]. Available: https://doi.org/10.1007/ s10846-019-01088-w

9. Ruggiero, F., Lippiello, V., Ollero, A.: Aerial Manipulation: A Literature Review. IEEE Robotics and Automation Letters 3(3), 1957-1964 (2018)

10. Bonyan Khamseh, H., Janabi-Sharifi, F., Abdessameud, A.: "Aerial Manipulation-A Literature Survey," Robotics and Autonomous Systems, vol. 107, pp. 221-235 (2018)

11. Alothman, Y., Gu, D.: "Quadrotor Transporting CableSuspended Load Using Iterative Linear Quadratic Regulator (iLQR) Optimal Control," in, : 8th Computer Science and Electronic Engineering (CEEC). Colchester, UK 2016, 168173 (2016)

12. Xian, B., Wang, S., Yang, S.: Nonlinear Adaptive Control for an Unmanned Aerial Payload Transportation System: Theory and Experimental Validation. Nonlinear Dynamics 98(3), 1745-1760 (2019)

13. Yang, S., Xian, B.: Energy-Based Nonlinear Adaptive Control Design for the Quadrotor UAV System With a Suspended Payload. IEEE Transactions on Industrial Electronics 67(3), 2054-2064 (2020)

14. Trachte, J., Gonzalez, F., McFadyen, A.: "Nonlinear Model Predictive Control for a Multi-Rotor with Heavy Slung Load," in 2014 International Conference on Unmanned Aircraft Systems (ICUAS), Orlando, FL, USA, pp. 1105-1110 (2014)

15. Yang, S., Xian, B.: "Robust Control Design for the Quadrotor UAV with a Suspended Payload," in 2018 IEEE 8th Annual International Conference on CYBER Technology in Automation, Control, and Intelligent Systems (CYBER), Tianjin, China, pp. 469-473 (2018)

16. de Angelis, E.L., Giulietti, F., Pipeleers, G.: Two-TimeScale Control of a Multirotor Aircraft for Suspended Load Transportation. Aerospace Science and Technology 84, 193-203 (2019)

17. Hua, H., Fang, Y., Zhang, X., Qian, C.: "Auto-tuning nonlinear pid-type controller for rotorcraft-based aggressive transportation," Mechanical Systems and Signal Processing, vol. 145, p. 106858 (2020) [Online]. Available: http://www. sciencedirect.com/science/article/pii/S0888327020302442

18. Palunko, I., Fierro, R., Cruz, P.: "Trajectory Generation for Swing-Free Maneuvers of a Quadrotor with Suspended Payload: A Dynamic Programming Approach,” in 2012 IEEE International Conference on Robotics and Automation, Saint Paul, MN, USA, pp. 2691-2697 (2012)

19. Wang, S., Xian, B.: "An Anti-Swing Trajectory Approach for an Unmanned Aerial Vehicle with A Slung Payload," in, : 37th Chinese Control Conference (CCC). Wuhan, China 2018, 5560-5565 (2018)
20. Geisert, M., Mansard, N.: "Trajectory Generation for Quadrotor Based Systems Using Numerical Optimal Control," in 2016 IEEE International Conference on Robotics and Automation (ICRA), Stockholm, Sweden, pp. 29582964 (2016)

21. Mellinger, D., Kumar, V.: "Minimum Snap Trajectory Generation and Control for Quadrotors," in 2011 IEEE International Conference on Robotics and Automation, Shanghai, China, pp. 2520-2525 (2011)

22. Sreenath, K., Michael, N., Kumar, V.: "Trajectory Generation and Control of a Quadrotor with a Cable-Suspended Load - A Differentially-Flat Hybrid System," in 2013 IEEE International Conference on Robotics and Automation, Karlsruhe, Germany, pp. 4888-4895 (2013)

23. Tang, S., Sreenath, K., Kumar, V.: "Aggressive Maneuvering of a Quadrotor with a Cable-Suspended Payload," in Robotics: Science and Systems. Workshop on Women in Robotics, California, USA (2014)

24. Richter, C., Bry, A., Roy, N.: "Polynomial Trajectory Planning for Aggressive Quadrotor Flight in Dense Indoor Environments," in Robotics Research: The 16th International Symposium ISRR, pp. 649-666. Springer International Publishing, Cham (2016)

25. Tang, S., Wüest, V., Kumar, V.: Aggressive Flight with Suspended Payloads Using Vision-Based Control. IEEE Robotics and Automation Letters 3(2), 1152-1159 (2018)

26. Liang, X., Fang, Y., Sun, N., Lin, H.: Dynamics Analysis and Time-Optimal Motion Planning for Unmanned Quadrotor Transportation Systems. Mechatronics 50, 16-29 (2018)

27. Sun, B., HU, C., Cao, L., Wang, N., Zhou, Y.: “Trajectory Planning of Quadrotor UAV with Suspended Payload Based on Predictive Control," in 2018 37th Chinese Control Conference (CCC), Wuhan, China, pp. 10 049-10 054 (2018)

28. Faust, A., Palunko, I., Cruz, P., Fierro, R., Tapia, L.: "Learning Swing-Free Trajectories for UAVs with a Suspended Load," in 2013 IEEE International Conference on Robotics and Automation, Karlsruhe, Germany, pp. 49024909 (2013)

29. Palunko, I., Faust, A., Cruz, P., Tapia, L., Fierro, R.: “A Reinforcement Learning Approach Towards Autonomous Suspended Load Manipulation Using Aerial Robots," in 2013 IEEE International Conference on Robotics and Automation, Karlsruhe, Germany, pp. 4896-4901 (2013)

30. Kim, S.-K., Ahn, C. K.: "Velocity-Sensorless ProportionalDerivative Trajectory Tracking Control with Active Damping for Quadcopters," Nonlinear Dynamics, vol. 103, no. 2, pp. 1681-1692 (2021) [Online]. Available: https://doi.org/ 10.1007/s11071-020-06193-2

31. Glida, H. E., Abdou, L., Chelihi, A., Sentouh, C., Hasseni, S.-E.-I.: "Optimal Model-Free Backstepping Control for a Quadrotor Helicopter,' Nonlinear Dynamics, vol. 100, no. 4, pp. 3449-3468 (2020) [Online]. Available: https://doi.org/ 10.1007/s11071-020-05671-x

32. Piedrafita, R., Comín, D., Beltrán, J.R.: Simulink® Implementation and Industrial Test of Input Shaping Techniques. Control Engineering Practice 79, 1-21 (2018)

33. Mohammed, A., Alghanim, K., Taheri Andani, M.: "An Adjustable Zero Vibration Input Shaping Control Scheme for Overhead Crane Systems," Shock and Vibration, vol. 2020, p. 7879839 (2020) 
34. Flash, T., Hogan, N.: The Coordination of Arm Movements: An Experimentally Confirmed Mathematical Model. The Journal of Neuroscience 5(7), 1688-1703 (1985)

35. Béarée, R., Olabi, A.: Dissociated Jerk-Limited Trajectory Applied to Time-Varying Vibration Reduction. Robotics and Computer-Integrated Manufacturing 29(2), 444-453 (2013)

36. Alzaydi, A.: "Time-optimal, minimum-jerk, and acceleration continuous looping and stitching trajectory generation for 5-axis on-the-fly laser drilling," Mechanical Systems and Signal Processing, vol. 121, pp. 532 - 550 (2019) [Online]. Available: http://www.sciencedirect.com/science/ article/pii/S0888327018307647

37. Oliveira, P.W., Barreto, G.A., Thé, G.A.P.: A General Framework for Optimal Tuning of PID-like Controllers for Minimum Jerk Robotic Trajectories. Journal of Intelligent \& Robotic Systems (2020)

38. Qian, L., Graham, S., Liu, H.H.: Guidance and Control Law Design for a Slung Payload in Autonomous Landing: A Drone Delivery Case Study. IEEE/ASME Transactions on Mechatronics 25(4), 1773-1782 (2020)

39. Qian, L., Liu, H. H.: "Dynamics and Control of a Quadrotor with a Cable Suspended Payload," in 2017 IEEE 30th Cana- dian Conference on Electrical and Computer Engineering (CCECE), pp. 1-4 (2017)

40. Zeng, J., Sreenath, K.: "Geometric Control of a Quadrotor with a Load Suspended from an Offset," in. American Control Conference (ACC) 2019, 3044-3050 (2019)

41. Richardson, M.J.E., Flash, T.: Comparing Smooth Arm Movements with the Two-Thirds Power Law and the Related Segmented-Control Hypothesis. The Journal of Neuroscience 22(18), 8201-8211 (2002)

42. Rao, S.S.: Mechanical Vibrations, 6th edn. Pearson, Hoboken (2017)

43. Piersol, A.G., Paez, T.L.: Harris' Shock and Vibration Handbook, 6th edn. McGraw-Hill, New York (2010)

44. Brunton, S.L., Kutz, J.N.: Data-Driven Science and Engineering: Machine Learning, Dynamical Systems, and Control. Cambridge University Press, (2019)

Publisher's Note Springer Nature remains neutral with regard to jurisdictional claims in published maps and institutional affiliations. 